\title{
Investigating the Hybrid Textures of Neutrino Mass Matrix for Near Maximal Atmospheric Neutrino Mixing
}

\author{
Madan Singh \\ Department of Physics, National Institute of Technology, Kurukshetra, Haryana 136119, India \\ Correspondence should be addressed to Madan Singh; singhmadan179@gmail.com
}

Received 22 December 2017; Accepted 5 April 2018; Published 14 May 2018

Academic Editor: Jose W. F. Valle

Copyright ( 2018 Madan Singh. This is an open access article distributed under the Creative Commons Attribution License, which permits unrestricted use, distribution, and reproduction in any medium, provided the original work is properly cited. The publication of this article was funded by $\mathrm{SCOAP}^{3}$.

\begin{abstract}
We have studied that the implication of a large value of the effective Majorana neutrino mass in case of neutrino mass matrices has either two equal elements and one zero element (popularly known as hybrid texture) or two equal cofactors and one zero minor (popularly known as inverse hybrid texture) in the flavor basis. In each of these cases, four out of sixty phenomenologically possible patterns predict near maximal atmospheric neutrino mixing angle in the limit of large effective Majorana neutrino mass. This feature remains irrespective of the experimental data on solar and reactor mixing angles. In addition, we have also performed the comparative study of all the viable cases of hybrid and inverse hybrid textures at $3 \sigma$ CL.
\end{abstract}

\section{Introduction}

In leptonic sector, the reactor mixing angle $\left(\theta_{13}\right)$ has been established to a reasonably good degree of precision [16], and its nonzero and relatively large value has not only provided an opportunity in exploring $\mathrm{CP}$ violation and the neutrino mass ordering in the future experiments but has also highlighted the puzzle of neutrino mass and mixing pattern. In spite of the significant developments made over the years, there are still several intriguing questions in the neutrino sector which remain unsettled. For instance, the present available data is unable to throw any light on the neutrino mass spectrum, which may be normal/inverted and may even be degenerate. Another important issue is the determination of octant of atmospheric mixing angle $\theta_{23}$, which may be greater than or less than or equal to $45^{\circ}$. The determination of the nature of neutrinos whether Dirac or Majorana also remains an open question. The observation of neutrinoless double beta $(0 \nu \beta \beta)$ decay would eventually establish the Majorana nature of neutrinos.

The effective Majorana mass term related to $0 v \beta \beta$ decay can be expressed as

$$
|M|_{e e}=\left|m_{1} c_{12}^{2} c_{13}^{2} e^{2 i \rho}+m_{2} s_{12}^{2} c_{13}^{2} e^{2 i \sigma}+m_{3} s_{13}^{2}\right| .
$$

Data from KamLAND-Zen experiment has presented an improved search for neutrinoless double-beta $(0 \nu \beta \beta)$ decay [7] and it is found that $|M|_{e e}<(0.061-0.165) \mathrm{eV}$ at $90 \%$ (or $<2 \sigma)$ CL. For recent reviews on $0 \nu \beta \beta$ decay see [8-13].

In the lack of any convincing theory, several phenomenological ideas have been proposed in the literature so as to restrict the form of neutrino mass matrix, such as some elements of neutrino mass matrix that are considered to be zero or equal [14-21] or some cofactors of neutrino mass matrix to be either zero or equal [19, 22-27]. Specifically, mass matrices with zero textures (or cofactors) have been extensively studied [14-18, 22-24] due to their connections to flavor symmetries. In addition, texture structures with one zero element (or minor) and an equality between two independent elements (or cofactors) in neutrino mass matrix have also been studied in the literature [20, 21, 26, 27]. Such form of texture structures sets to one constraint equation and thus reduces the number of real free parameters of neutrino mass matrix to seven. Hence they are considered as predictive as the well-known two-zero textures and can also be realised within the framework of seesaw mechanism. Out of sixty possibilities, only fifty-four are found to be compatible with the neutrino oscillation data [21] for texture structures having one zero element and equal matrix elements in the 
neutrino mass matrix (1TEE), while for texture with one vanishing minor and equal cofactors in the neutrino mass matrix (1TEC) only fifty-two cases are able to survive the data $[26,27]$.

The purpose of present paper is to investigate the implication of large effective neutrino mass $|M|_{e e}$ on 1TEE and 1TEC structures of neutrino mass matrix, while taking into account the assumptions of $[28,29]$. The consideration of large $|M|_{e e}$ is motivated by the extensive search for this parameter in the ongoing $0 v \beta \beta$ experiments. The implication of large $|M|_{e e}$ has earlier been studied for the viable cases of texture two-zero and two-vanishing minor, respectively $[28,29]$. Grimus et al. [30] also predicted the near maximal atmospheric mixing for two-zero textures when supplemented with the assumption of quasi-degenerate mass spectrum. However, the observation made in all these analyses is independent of solar and reactor mixing angles. Motivated by these works, we find that only four out of sixty cases are able to predict near maximal $\theta_{23}$ for 1TEE and 1TEC, respectively. In addition, the analysis also hints towards the indistinguishable feature of 1TEE and 1TEC. To present the indistinguishable nature of the 1TEE and 1TEC texture structures, we have then carried out a comparative study of all the viable cases of 1TEE and 1TEC at $3 \sigma$ CL. The similarity between texture zero structures with one mass ordering and corresponding cofactor zero structures with the opposite mass ordering has earlier been noted in [31-33]. In [19], the strong similarities have also been noted between the texture structures with two equalities of elements and structures with two equalities of cofactors in neutrino mass matrix, with opposite mass ordering.

The rest of the paper is planned in the following manner. In Section 2, we shall discuss the methodology to obtain the constraint equations. Section 3 is devoted to numerical analysis. In the end we will summarize our result.

\section{Methodology}

The effective Majorana neutrino mass matrix $\left(M_{v}\right)$ contains nine parameters which include three neutrino masses $\left(m_{1}\right.$, $\left.m_{2}, m_{3}\right)$, three mixing angles $\left(\theta_{12}, \theta_{23}, \theta_{13}\right)$, and three CP violating phases $(\delta, \rho, \sigma)$. In the flavor basis, the Majorana neutrino mass matrix can be expressed as follows:

$$
M_{\nu}=P_{l} U P_{v} M^{\text {diag }} P_{v}^{T} U^{T} P_{l}^{T},
$$

where $M^{\text {diag }}=\operatorname{diag}\left(m_{1}, m_{2}, m_{3}\right)$ is the diagonal matrix of neutrino masses and $U$ is the flavor mixing matrix, and

$$
\begin{aligned}
& P_{v}=\left(\begin{array}{ccc}
e^{i \rho} & 0 & 0 \\
0 & e^{i \sigma} & 0 \\
0 & 0 & 1
\end{array}\right), \\
& P_{l}=\left(\begin{array}{ccc}
e^{i \phi_{e}} & 0 & 0 \\
0 & e^{i \phi_{\mu}} & 0 \\
0 & 0 & e^{i \phi_{\tau}}
\end{array}\right),
\end{aligned}
$$

where $P_{v}$ is diagonal phase matrix containing Majorana neutrinos $\rho, \sigma . P_{l}$ is unobservable phase matrix and depends on phase convention. Equation (2) can be rewritten as

$$
M_{v}=P_{l} U\left(\begin{array}{ccc}
\lambda_{1} & 0 & 0 \\
0 & \lambda_{2} & 0 \\
0 & 0 & \lambda_{3}
\end{array}\right) U^{T} P_{l}^{T}
$$

where $\lambda_{1}=m_{1} e^{2 i \rho}, \lambda_{2}=m_{2} e^{2 i \sigma}, \lambda_{3}=m_{3}$. For the present analysis, we consider the following parameterization of $U$ [20]:

$U$

$$
=\left(\begin{array}{ccc}
c_{12} c_{13} & s_{12} c_{13} & s_{13} \\
-c_{12} s_{23} s_{13}-s_{12} c_{23} e^{-i \delta} & -s_{12} s_{23} s_{13}+c_{12} c_{23} e^{-i \delta} & s_{23} c_{13} \\
-c_{12} c_{23} s_{13}+s_{12} s_{23} e^{-i \delta} & -s_{12} c_{23} s_{13}-c_{12} s_{23} e^{-i \delta} & c_{23} c_{13}
\end{array}\right),
$$

where $c_{i j}=\cos \theta_{i j}, s_{i j}=\sin \theta_{i j}$. Here, $U$ is a $3 \times 3$ unitary matrix consisting of three flavor mixing angles $\left(\theta_{12}, \theta_{23}, \theta_{13}\right)$ and one Dirac CP-violating phase $\delta$.

For hybrid texture structure (1TEE) of $M_{\nu}$, we can express the ratios of neutrino mass eigenvalues in terms of the mixing matrix elements as [21]

$$
\begin{aligned}
& \frac{\lambda_{1}}{\lambda_{3}}=\frac{P\left(U_{a 3} U_{b 3} U_{\alpha 2} U_{\beta 2}-U_{a 2} U_{b 2} U_{\alpha 3} U_{\beta 3}\right)+\left(U_{a 2} U_{b 2} U_{c 3} U_{d 3}-U_{a 3} U_{b 3} U_{c 2} U_{d 2}\right)}{P\left(U_{a 2} U_{b 2} U_{\alpha 1} U_{\beta 1}-U_{a 1} U_{b 1} U_{\alpha 2} U_{\beta 2}\right)+\left(U_{a 1} U_{b 1} U_{c 2} U_{d 2}-U_{a 2} U_{b 2} U_{c 1} U_{d 1}\right)}, \\
& \frac{\lambda_{2}}{\lambda_{3}}=\frac{P\left(U_{a 1} U_{b 1} U_{\alpha 3} U_{\beta 3}-U_{a 3} U_{b 3} U_{\alpha 1} U_{\beta 1}\right)+\left(U_{a 3} U_{b 3} U_{c 1} U_{d 1}-U_{a 1} U_{b 1} U_{c 3} U_{d 3}\right)}{P\left(U_{a 2} U_{b 2} U_{\alpha 1} U_{\beta 1}-U_{a 1} U_{b 1} U_{\alpha 2} U_{\beta 2}\right)+\left(U_{a 1} U_{b 1} U_{c 2} U_{d 2}-U_{a 2} U_{b 2} U_{c 1} U_{d 1}\right)},
\end{aligned}
$$

where $P=e^{i\left(\phi_{\alpha}+\phi_{\beta}-\phi_{c}-\phi_{d}\right)}$ is a phase factor. Similarly, in case of inverse hybrid texture structure (1TEC) of $M_{\nu}$, we can express the ratios of mass eigenvalues as $[26,27]$ follows:

$$
\frac{\lambda_{1}}{\lambda_{3}}=\frac{A_{1} B_{2}-A_{2} B_{1}}{A_{2} B_{3}-A_{3} B_{2}},
$$

$$
\frac{\lambda_{2}}{\lambda_{3}}=\frac{A_{1} B_{2}-A_{2} B_{1}}{A_{3} B_{1}-A_{1} B_{3}},
$$

where

$$
A_{i}=\left(U_{p j} U_{q j} U_{r k} U_{s k}-U_{t j} U_{u j} U_{v k} U_{w k}\right)+(j \longleftrightarrow k),
$$




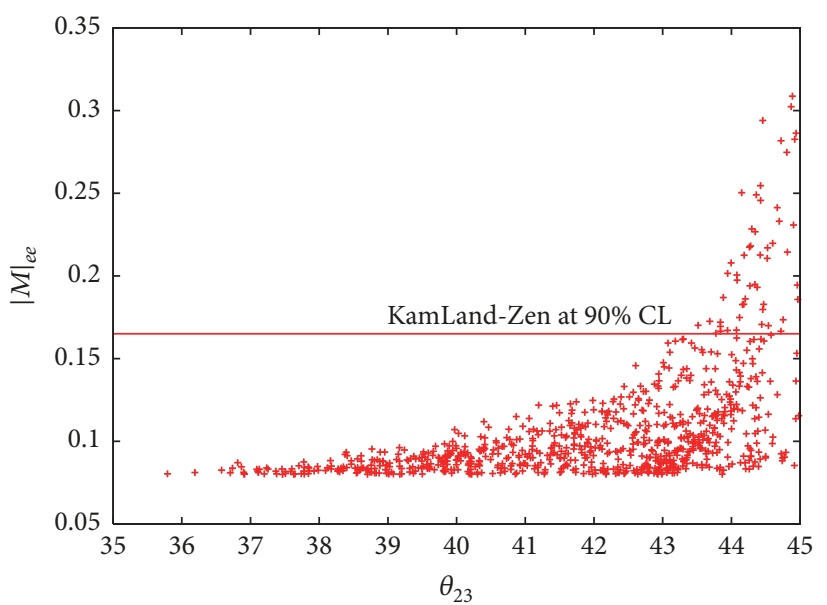

(a)

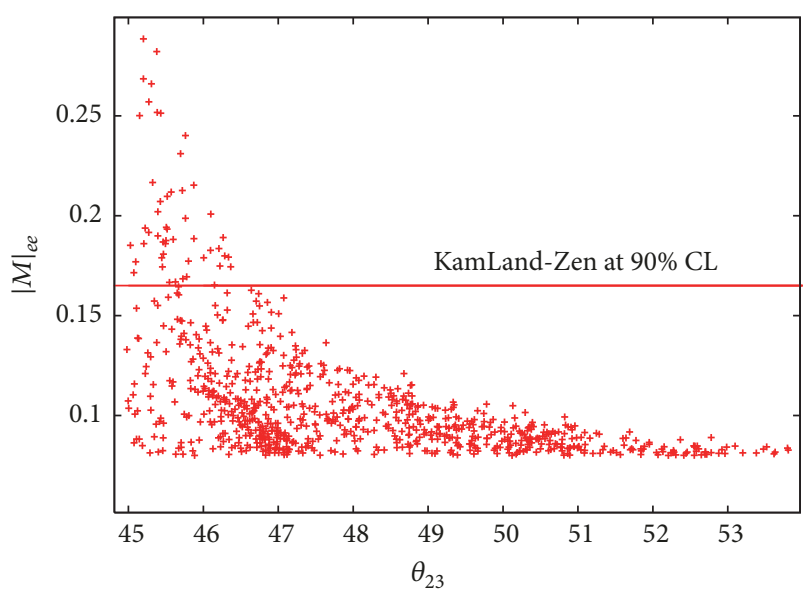

(c)

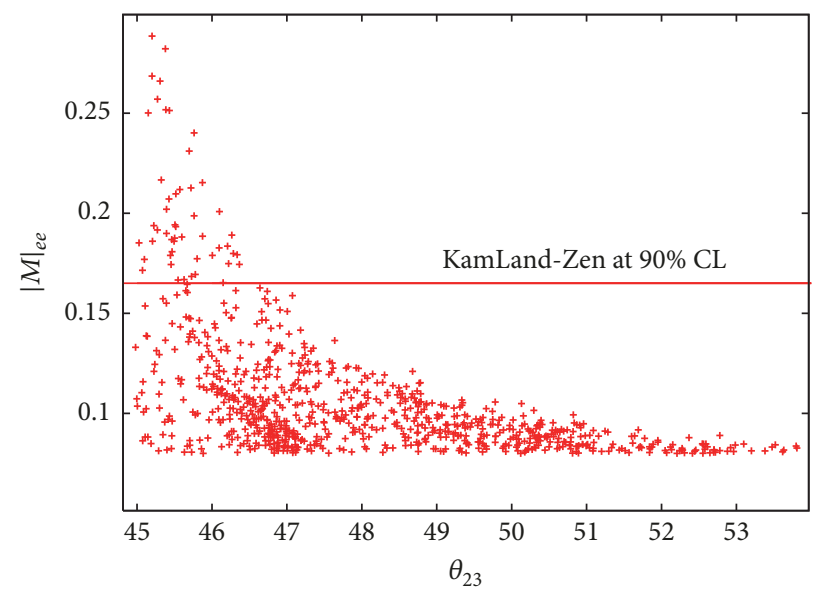

(b)

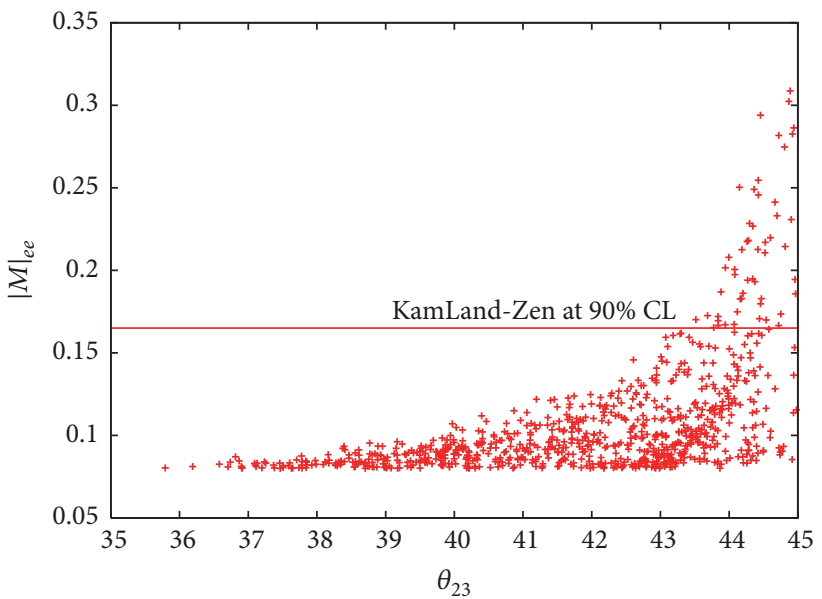

(d)

FIgure 1: Correlation plots for textures $B_{2}$ ((a) NO and (b) IO) and $C_{7}$ for ((c) NO and (d) IO) at $3 \sigma$ CL for 1TEE. The symbols have their usual meaning. The horizontal line indicates the upper limit on effective neutrino mass term $|M|_{e e}\left(\mathrm{i} . e .,|M|_{e e}<0.165 \mathrm{eV}\right)$ at $90 \% \mathrm{CL}$, given in KamLAND-Zen experiment [7].

$$
\begin{aligned}
& B_{i}=(-1)^{m+n} Q\left(U_{a j} U_{b j} U_{c k} U_{d k}-U_{e j} U_{f j} U_{g k} U_{h k}\right) \\
& -(-1)^{m^{\prime}+n^{\prime}}\left(U_{a^{\prime} j} U_{b^{\prime} j} U_{c^{\prime} k} U_{d^{\prime} k}-U_{e^{\prime} j} U_{f^{\prime} j} U_{g^{\prime} k} U_{h^{\prime} k}\right) \\
& \quad+(j \longleftrightarrow k),
\end{aligned}
$$

with $(i, j, k)$ a cyclic permutation of $(1,2,3)$ and $Q=$ $e^{i\left(\phi_{a}+\phi_{b}+\phi_{c}+\phi_{d}-\phi_{a^{\prime}}-\phi_{b^{\prime}}-\phi_{c^{\prime}}-\phi_{d^{\prime}}\right)}$ is phase factor.

Using the above expressions, we can obtain the magnitude of neutrino mass ratios, $\alpha \equiv\left|\lambda_{1}\right| /\left|\lambda_{3}\right|$ and $\beta \equiv\left|\lambda_{2}\right| /\left|\lambda_{3}\right|$ in each texture structure, and the Majorana phases $(\rho, \sigma)$ can be given as $\rho=(1 / 2) \arg \left(\lambda_{1} / \lambda_{3}\right)$ and $\sigma=(1 / 2) \arg \left(\lambda_{2} / \lambda_{3}\right)$.

The solar and atmospheric mass squared differences $\left(\delta m^{2}, \Delta m^{2}\right)$, where $\delta m^{2}$ corresponds to solar mass-squared difference and $\Delta m^{2}$ corresponds to atmospheric masssquared difference, can be defined as [20]

$$
\delta m^{2}=\left(m_{2}^{2}-m_{1}^{2}\right),
$$

$$
\Delta m^{2}=m_{3}^{2}-\frac{1}{2}\left(m_{1}^{2}+m_{2}^{2}\right) .
$$

The experimentally determined solar and atmospheric neutrino mass-squared differences can be related to neutrino mass ratios $(\alpha, \beta)$ as

$$
R_{\nu} \equiv \frac{\delta m^{2}}{\left|\Delta m^{2}\right|}=\frac{2\left(\beta^{2}-\alpha^{2}\right)}{\left|2-\left(\beta^{2}+\alpha^{2}\right)\right|},
$$

and the three neutrino masses can be determined in terms of $\alpha, \beta$ as

$$
\begin{aligned}
& m_{3}=\sqrt{\frac{\delta m^{2}}{\beta^{2}-\alpha^{2}}}, \\
& m_{2}=m_{3} \beta, \\
& m_{1}=m_{3} \alpha .
\end{aligned}
$$




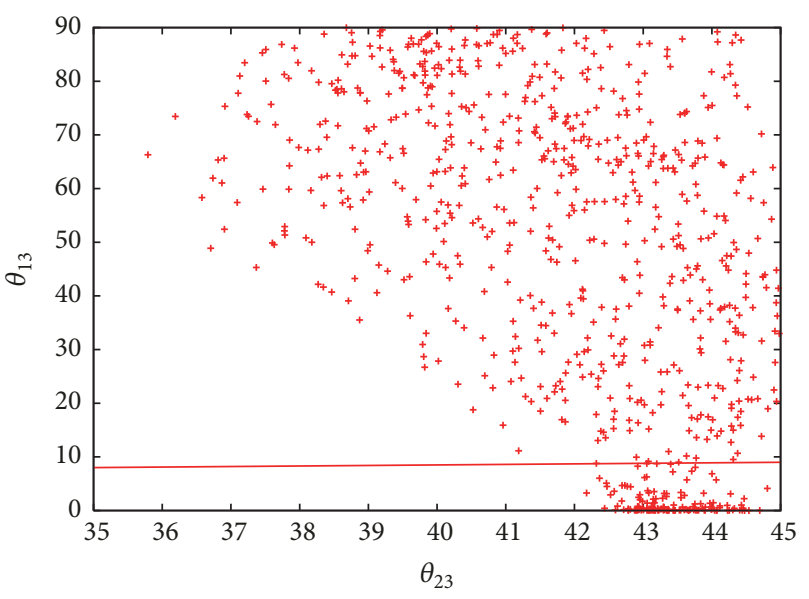

(a)

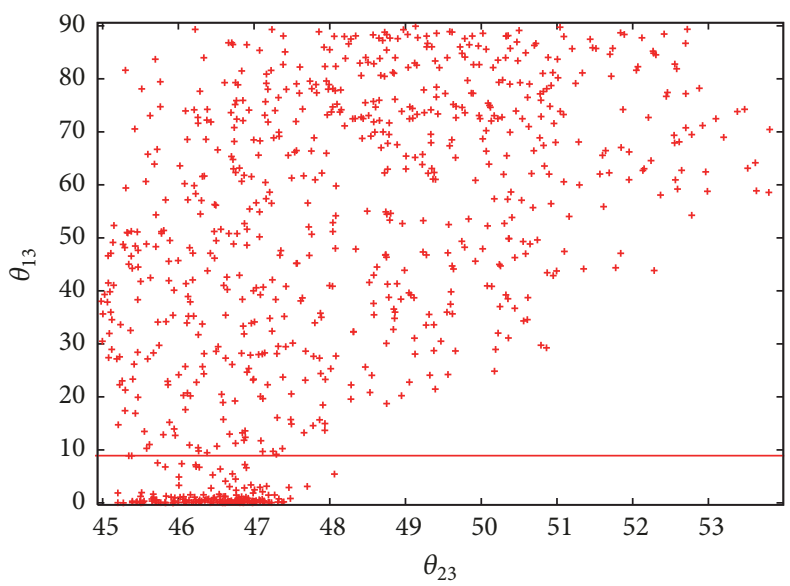

(c)

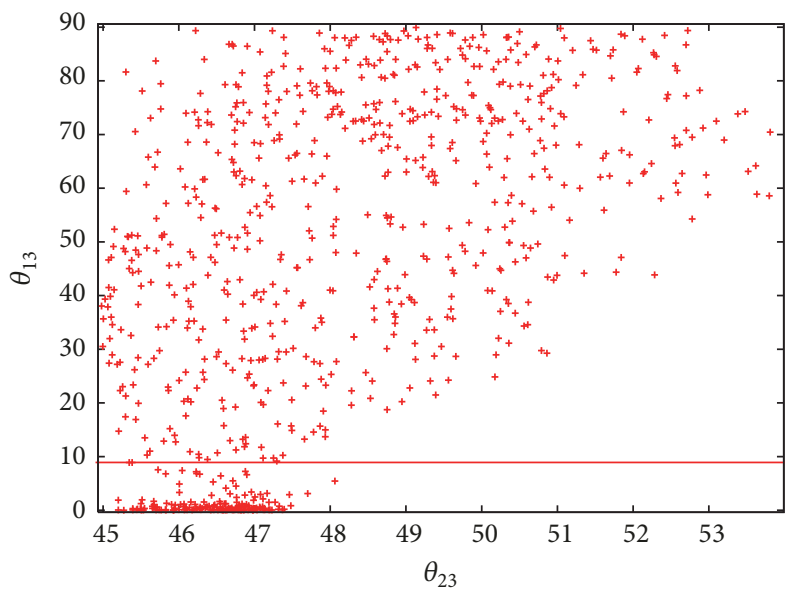

(b)

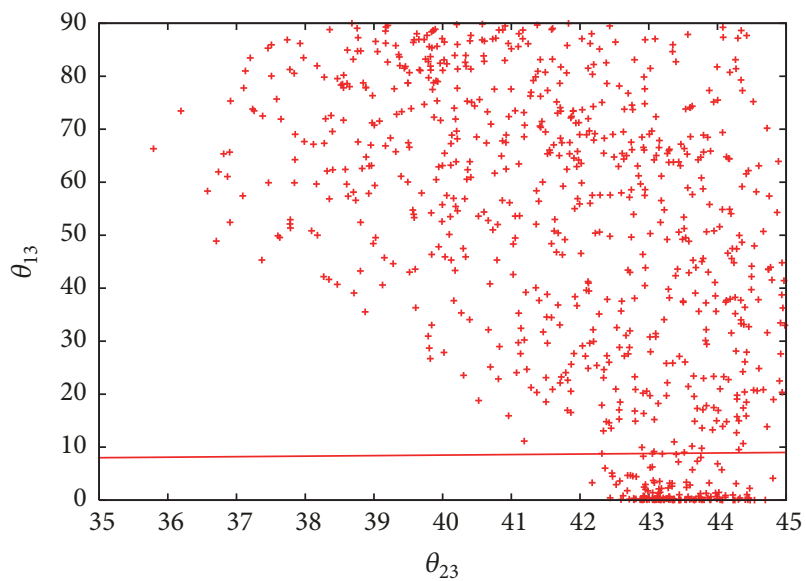

(d)

Figure 2: Correlation plots for textures $B_{2}$ ((a) NO and (b) IO) and $C_{7}$ for ((c) NO and (d) IO) at $3 \sigma$ CL for 1TEE. The symbols have their usual meaning. The horizontal line indicates the upper limit on reactor mixing angle $\theta_{13}<8.9^{0}$, as given in Table 1 .

Among the sixty logically possible cases of 1TEE or 1TEC texture structures, there are certain pair, which exhibit similar phenomenological implications and are related via permutation symmetry [21, 26, 27]. This corresponds to permutation of the 2-3 rows and 2-3 columns of $M_{\nu}$. The corresponding permutation matrix can be given by

$$
P_{23}=\left(\begin{array}{lll}
1 & 0 & 0 \\
0 & 0 & 1 \\
0 & 1 & 0
\end{array}\right) .
$$

With the help of permutation symmetry, one obtains the following relations among the neutrino oscillation parameters:

$$
\begin{aligned}
& \theta_{12}^{X}=\theta_{12}^{Y}, \\
& \theta_{23}^{X}=90^{\circ}-\theta_{23}^{Y}, \\
& \theta_{13}^{X}=\theta_{13}^{Y}, \\
& \delta^{X}=\delta^{Y}-180^{\circ},
\end{aligned}
$$

where $X$ and $Y$ denote the cases related to 2-3 permutation. The following pair among sixty cases are related via permutation symmetry:

$$
\begin{aligned}
& \left(A_{1}, A_{1}\right) ;\left(A_{2}, A_{8}\right) ;\left(A_{3}, A_{7}\right) ;\left(A_{4}, A_{6}\right) ;\left(A_{5}, A_{5}\right) ; \\
& \left(A_{9}, A_{10}\right) ;\left(B_{1}, C_{1}\right) \\
& \left(B_{2}, C_{7}\right) ;\left(B_{3}, C_{6}\right) ;\left(B_{4}, C_{5}\right) ;\left(B_{5}, C_{4}\right) ; \\
& \left(B_{6}, C_{3}\right)\left(B_{7}, C_{2}\right) ;\left(B_{8}, C_{10}\right) \\
& \left(B_{9}, C_{9}\right) ;\left(B_{10}, C_{8}\right) ;\left(D_{1}, F_{2}\right) ;\left(D_{2}, F_{1}\right) ;\left(D_{3}, F_{4}\right) ; \\
& \left(D_{4}, F_{3}\right) \\
& \left(D_{5}, F_{5}\right) ;\left(D_{6}, F_{9}\right) ;\left(D_{7}, F_{8}\right) ;\left(D_{8}, F_{7}\right) ;\left(D_{9}, F_{6}\right) ; \\
& \left(D_{10}, F_{10}\right) \\
& \left(E_{1}, E_{2}\right) ;\left(E_{3}, E_{4}\right) ;\left(E_{5}, E_{5}\right) ;\left(E_{6}, E_{9}\right) ;\left(E_{7}, E_{8}\right) ; \\
& \left(E_{10}, E_{10}\right) .
\end{aligned}
$$

Clearly we are left with only thirty-two independent cases. It is worthwhile to mention that cases $A_{1}, A_{5}, E_{5}$, and $E_{10}$ are invariant under the permutations of 2 and 3 rows and columns. 


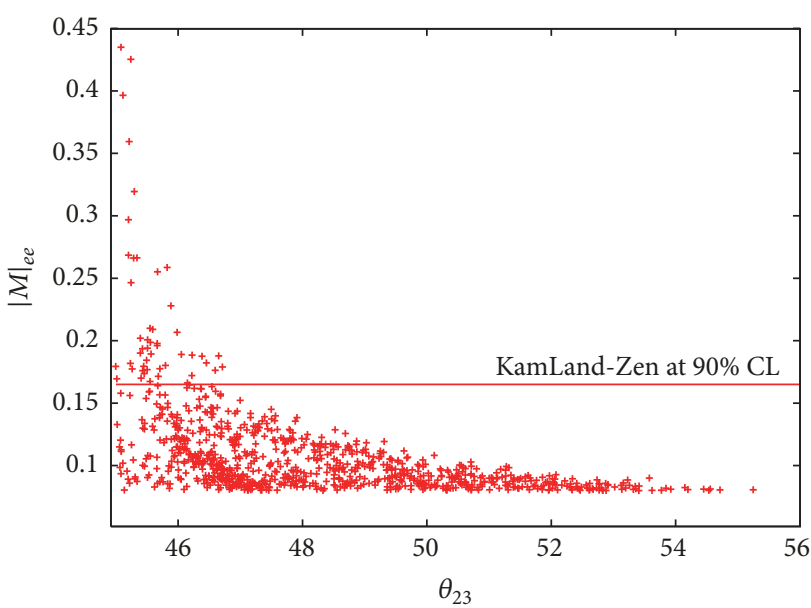

(a)

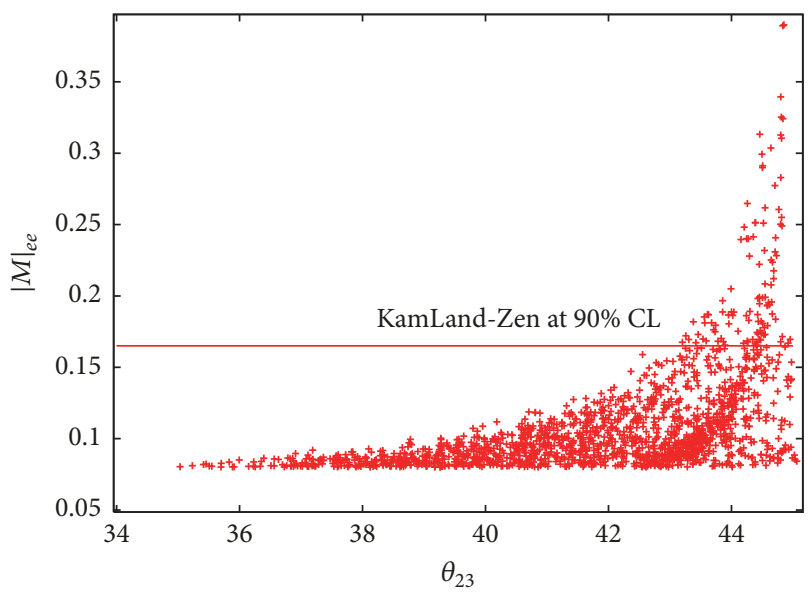

(c)

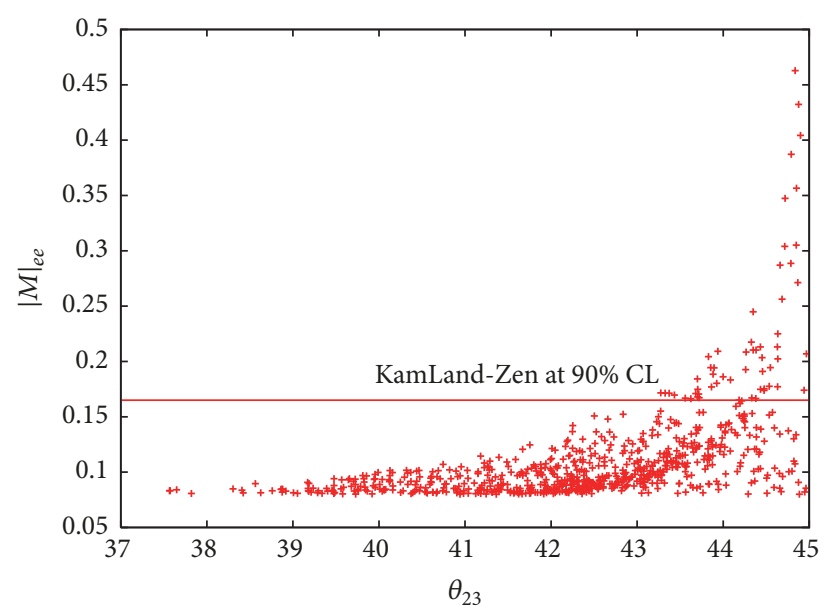

(b)

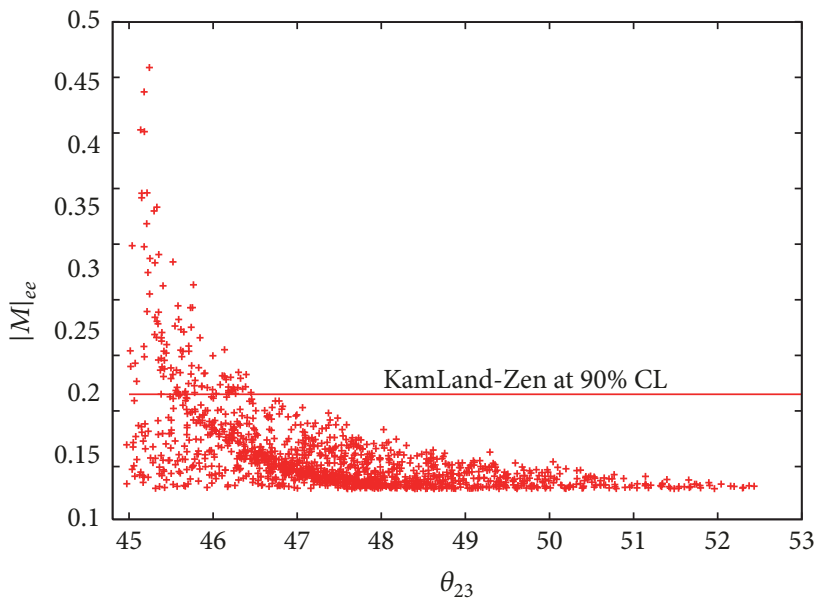

(d)

Figure 3: Correlation plots for textures $B_{2}$ ((a) NO and (b) IO) and $C_{7}$ for ((c) NO and (d) IO) at $3 \sigma$ CL for 1TEC. The symbols have their usual meaning. The horizontal line indicates the upper limit on effective neutrino mass term $|M|_{e e}\left(\right.$ i.e., $\left.|M|_{e e}<0.165 \mathrm{eV}\right)$ at $90 \%(<2 \sigma) \mathrm{CL}$, given in KamLAND-Zen experiment [7].

\section{Numerical Analysis}

The experimental constraints on neutrino parameters at $3 \sigma$ confidence levels (CL) are given in Table 1. The classification of sixty phenomenologically possible cases of 1TEE and 1TEC is done in the nomenclature, given by Wang et al. in [26, 27]. All the sixty cases are divided into six categories $A, B, C, D$, and $E$ (Table 2). In $[26,27]$, it is found that the phenomenological results of cases belonging to 1TEC (or 1TEE) are almost similar to each other due to permutation symmetry. For the purpose of calculation, we have used the latest experimental data on neutrino mixing angles $\left(\theta_{12}, \theta_{23}, \theta_{13}, \delta m^{2}\right)$ and mass squared differences $\left(\Delta m^{2}, \delta\right)$ at $3 \sigma \mathrm{CL}[5,6]$.

3.1. Near Maximal Atmospheric Mixing for ITEE and ITEC Texture Structures. As a first step of the analysis, all the sixty cases of ITEE and ITEC have been investigated in the limit of large $|M|_{e e}$. For the analysis, we have incorporated the assumptions of $[28,29]$, wherein authors have considered the lower bound on $|M|_{e e}$ to be large (i.e., $|M|_{e e}>0.08 \mathrm{eV}$ ). The upper bound on $|M|_{e e}$ is chosen to be more conservative; that is, $|M|_{e e}<0.5 \mathrm{eV}$ at $3 \sigma \mathrm{CL}$ [10]. The input parameters $\left(\theta_{12}, \theta_{23}, \theta_{13}, \delta m^{2}, \Delta m^{2}, \delta\right)$ are generated by the method of random number generation. The three neutrino mixing angles and Dirac-type CP-violating phase $\delta$ are varied between $0^{0}$ to $90^{\circ}$ and $0^{0}$ to $360^{\circ}$, respectively. However, the mass-squared differences $\left(\delta m^{2}, \Delta m^{2}\right)$ are varied randomly within their $3 \sigma$ experimental range $[5,6]$. For the numerical analysis, we follow the same procedure as discussed in [20]. The main results and discussion are summarized as follows.

In Figures $1,2,3,4,5$, and 6, it is explicitly shown that the octant of $\theta_{23}$ is well restricted for $B_{2}, C_{7}, D_{3}, F_{4}$ of 1TEE and ITEC texture structures, respectively. However, for the remaining cases, the value of $\theta_{23}$ is unconstrained like other oscillation parameters. Apart from restricting the octant of $\theta_{23}$, the analysis also ensures the quasi-degenerate mass ordering for these cases similar to the observation of [2830]. From Figures 1(a), 1(b), 3(a), and 3(b), it is clear that, 


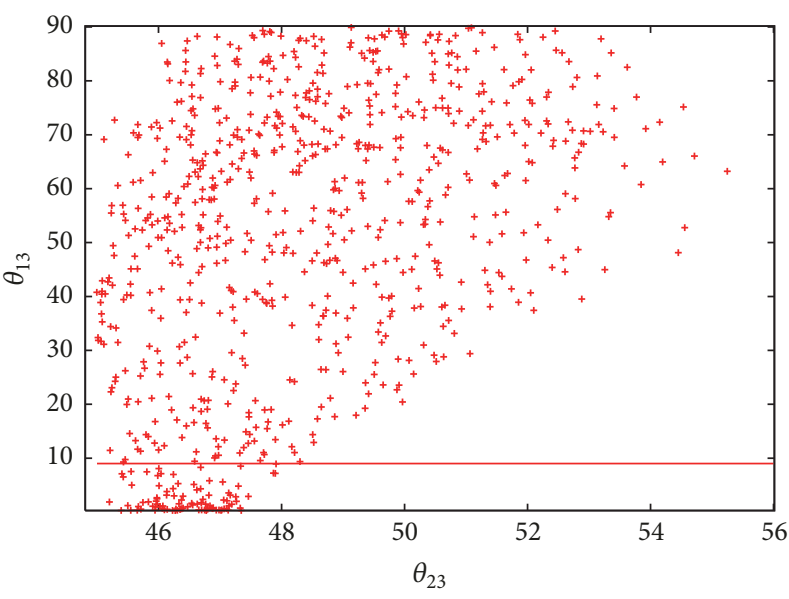

(a)

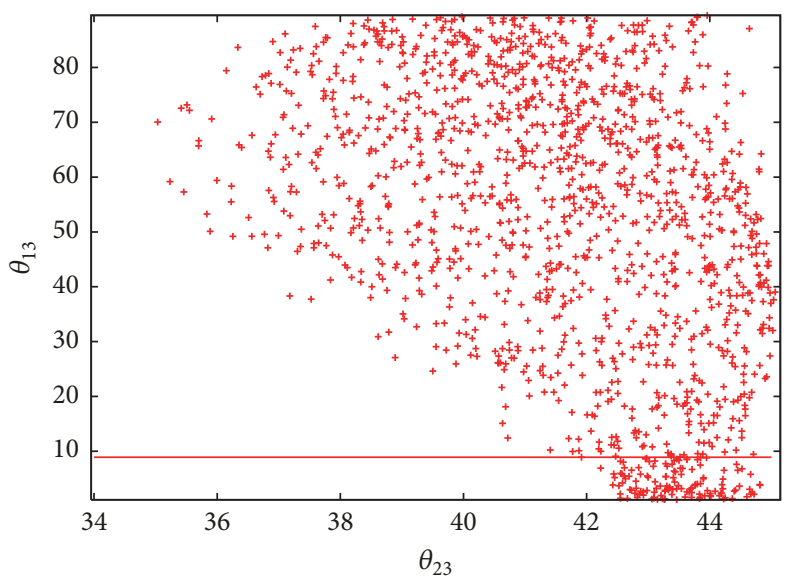

(c)

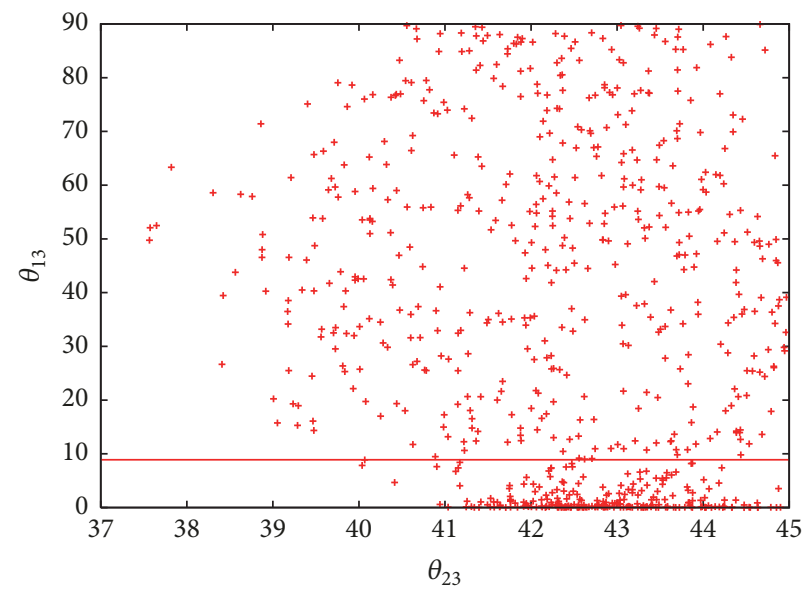

(b)

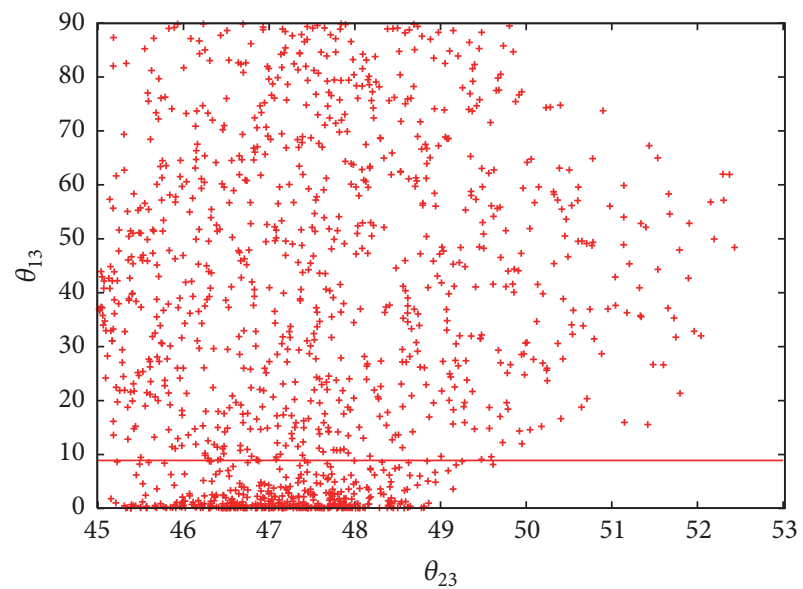

(d)

Figure 4: Correlation plots for textures $B_{2}$ ((a) NO and (b) IO) and $C_{7}$ for ((c) NO and (d) IO) at $3 \sigma$ CL for 1TEC. The symbols have their usual meaning. The horizontal line indicates the upper limit on reactor mixing angle $\theta_{13}<8.9^{0}$, as given in Table 1 .

TABLE 1: Current neutrino oscillation parameters from global fits at $3 \sigma$ confidence level $[5,6]$. NO (IO) refers to normal (inverted) neutrino mass ordering.

\begin{tabular}{lcc}
\hline Parameter & Best fit & $3 \sigma$ \\
\hline$\delta m^{2}\left[10^{-5} \mathrm{eV}^{2}\right]$ & 7.50 & $7.03-8.09$ \\
$\left|\Delta m_{31}^{2}\right|\left[10^{-3} \mathrm{eV}^{2}\right](\mathrm{NO})$ & 2.52 & $2.407-2.643$ \\
$\left|\Delta m_{31}^{2}\right|\left[10^{-3} \mathrm{eV}^{2}\right](\mathrm{IO})$ & 2.52 & $2.39-2.63$ \\
$\theta_{12}$ & $33.56^{\circ}$ & $31.3^{\circ}-35.99^{\circ}$ \\
$\theta_{23}(\mathrm{NO})$ & $41.6^{\circ}$ & $38.4^{\circ}-52.8^{\circ}$ \\
$\theta_{23}(\mathrm{IO})$ & $50.0^{\circ}$ & $38.8^{\circ}-53.1^{\circ}$ \\
$\theta_{13}(\mathrm{NO})$ & $8.46^{\circ}$ & $7.99^{\circ}-8.90^{\circ}$ \\
$\theta_{13}(\mathrm{IO})$ & $8.49^{\circ}$ & $8.03^{\circ}-8.93^{\circ}$ \\
$\delta(\mathrm{NO})$ & $261^{\circ}$ & $0^{\circ}-360^{\circ}$ \\
$\delta(\mathrm{IO})$ & $277^{\circ}$ & $145^{\circ}-391^{\circ}$ \\
\hline
\end{tabular}

for increasing value of $|M|_{e e}$, atmospheric mixing angle $\theta_{23}$ approaches to maximal value for the structure $B_{2}$ of 1TEE and 1TEC for both normal ordering (NO) and inverted ordering (IO). In Figures 2 and 4, it is explicitly shown that for cases $B_{2}$ and $C_{7}$ the quadrant of $\theta_{23}$ is already decided without the experimental input of the mixing angles. For 1TEE, we have $\theta_{23}<45^{\circ}$ for NO and $\theta_{23}>45^{\circ}$ for IO, whereas for 1TEC, $\theta_{23}>45^{\circ}$ for NO, while $\theta_{23}<45^{\circ}$ for IO (Figures 2(a), 2(b), $4(\mathrm{a})$, and $4(\mathrm{~b}))$. Clearly the correlation plots of case $B_{2}$ are indistinguishable for 1TEE and 1TEC, if neutrino mass 


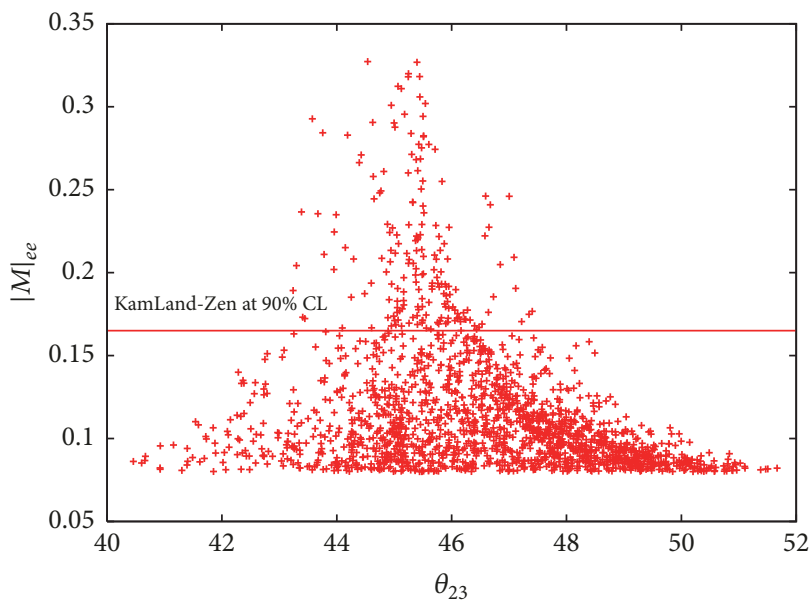

(a)

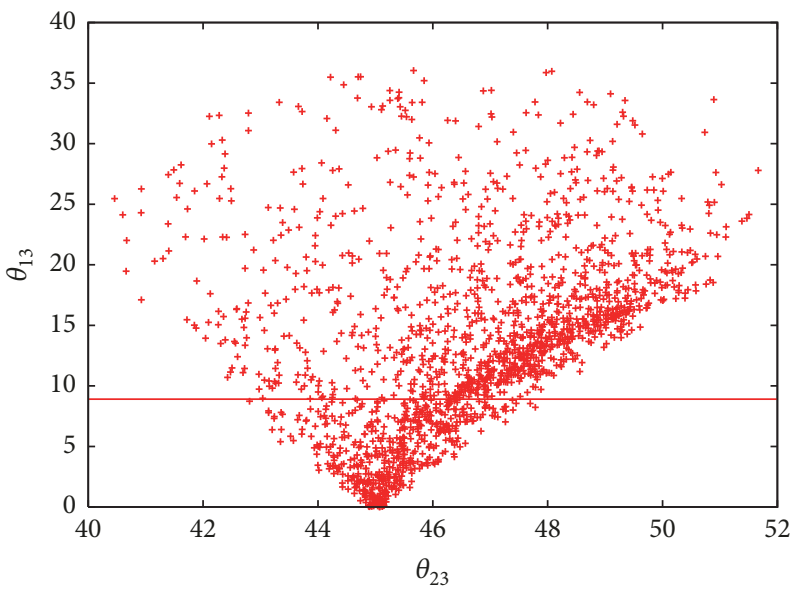

(c)

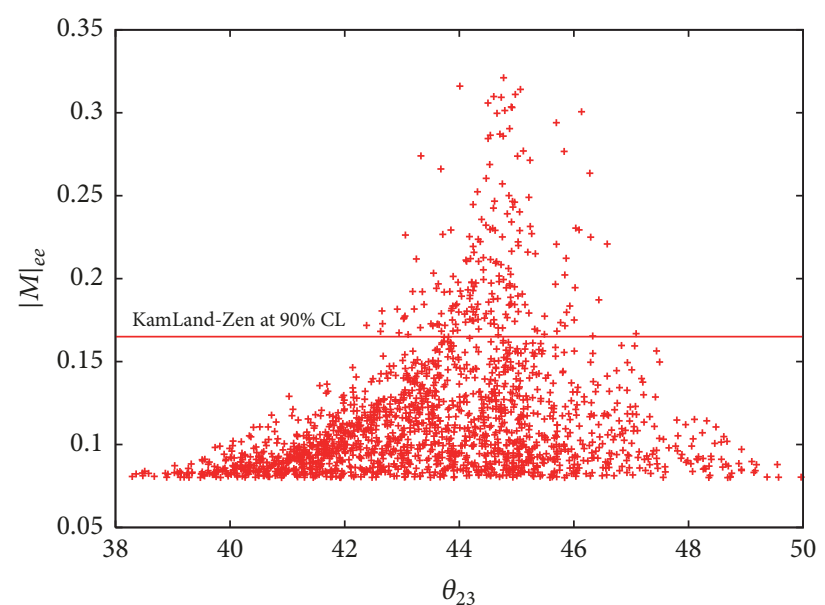

(b)

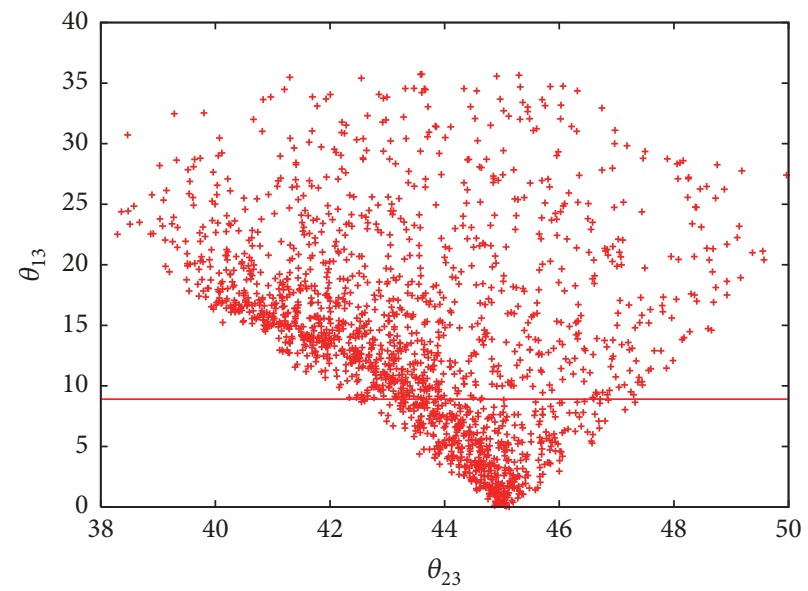

(d)

FIGURE 5: Correlation plots for textures $D_{3}((\mathrm{a}),(\mathrm{c}))$ and $F_{4}((\mathrm{~b}),(\mathrm{d}))$ with IO for 1TEE at $3 \sigma \mathrm{CL}$. The symbols have their usual meaning. In (a) and (b), colored horizontal line indicates the upper limit on effective neutrino mass term $|M|_{e e}$ (i.e., $\left.|M|_{e e}<0.165 \mathrm{eV}\right)$ at $90(<2 \sigma) \% \mathrm{CL}$, given in KamLAND-Zen experiment [7]. In (c) and (d), we have shown the upper limit on reactor mixing angle $\theta_{13}$.

ordering is not considered as also pointed out earlier. Similar conclusion can be drawn for structure $C_{7}$ since both are related through 2-3 exchange symmetry (Figures $2(\mathrm{c}), 2(\mathrm{~d})$, $4(\mathrm{c})$, and 4(d)). Apart from the prediction of near maximality of $\theta_{23}$, cases $B_{2}$ and $C_{7}$ also predict $\delta \simeq 90^{\circ}, 270^{\circ}$ for ITEE and 1TEC, respectively, if experimental range of mixing angles is considered as in Table 2. Figures 2(a) and 2(c) for NO and Figures 2(b) and 2(d) for IO depict the 2-3 interchange symmetry between cases $B_{2}$ and $C_{7}$ for 1TEE. Similar phenomenological observation is shown for 1TEC in Figures 4(a), 4(c), 4(b), and 4(d), respectively.

Similarly, cases $\mathrm{D}_{3}$ and $F_{4}$ of 1TEE also predict near maximal atmospheric mixing angle $\left(\theta_{23}\right)$ for IO (Figures $5(\mathrm{a})$ and 5(b)). Interestingly the parameter space of reactor mixing angle $\theta_{13}$ is found to be constrained between $0^{0}$ and $35^{\circ}$ (Figures 5(c) and 5(d)). In Figures 5(c) and 5(d), it is clear that, for the allowed experimental range of $\theta_{13}\left(8.5^{0}-9.8^{0}\right), \theta_{23}$ inches closer to $45^{\circ}$. Similar predictions have been noted for cases $D_{3}$ and $F_{4}$ of 1TEC, however, for normal mass ordering (NO) (Figures 6(a), 6(b), 6(c), and 6(d)).
3.2. Comparing the Results for 1TEE and ITEC Texture Structures. In this subsection, we compare the results of all the viable structures of 1TEE and 1TEC in neutrino mass matrix. It is worthwhile to mention that the present refinements of the experimental data do not limit the number of viable cases in 1TEE and 1TEC textures respectively. The number of viable cases obtained is the same as predicted in [21, $26,27]$ for 1 TEE and ITEC, respectively. For executing the analysis, we vary the allowed ranges of three neutrino mixing angles $\left(\theta_{12}, \theta_{23}, \theta_{13}\right)$ and mass squared differences $\left(\delta m^{2}, \Delta m^{2}\right)$ within their $3 \sigma$ confidence level. To facilitate the comparison, we have encapsulated the the predictions regarding three $\mathrm{CP}$ violating phases $(\rho, \sigma, \delta)$ and neutrino masses $m_{1,2,3}$ for all the allowed texture structures of 1TEE and 1TEC, respectively (Tables 3, 4, 5, and 6).

Category A. In Category A, there are 10 possible cases out of which only four $\left(A_{1,4,5,6}\right)$ are allowed for 1TEE at $3 \sigma \mathrm{CL}$, and in addition inverted mass ordering (IO) is ruled out for all these cases. On the other hand, only three $\left(A_{1,4,6}\right)$ are allowed 


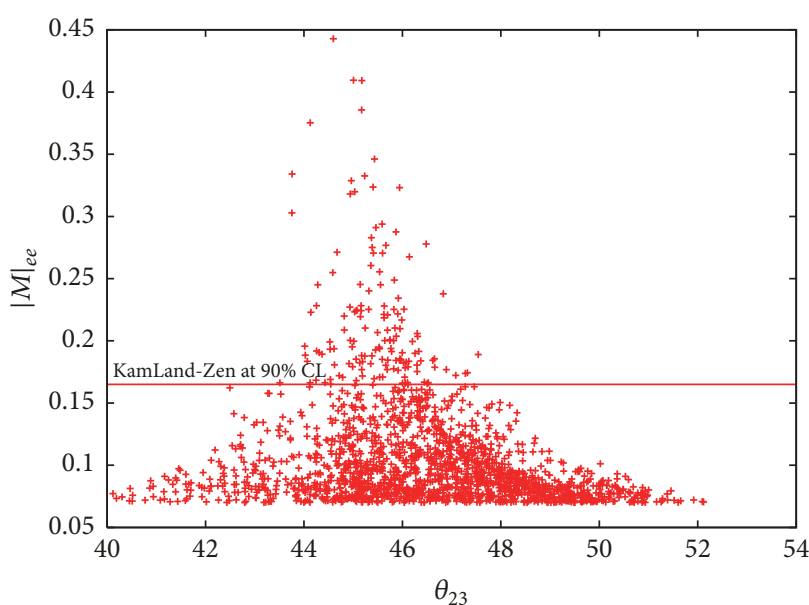

(a)

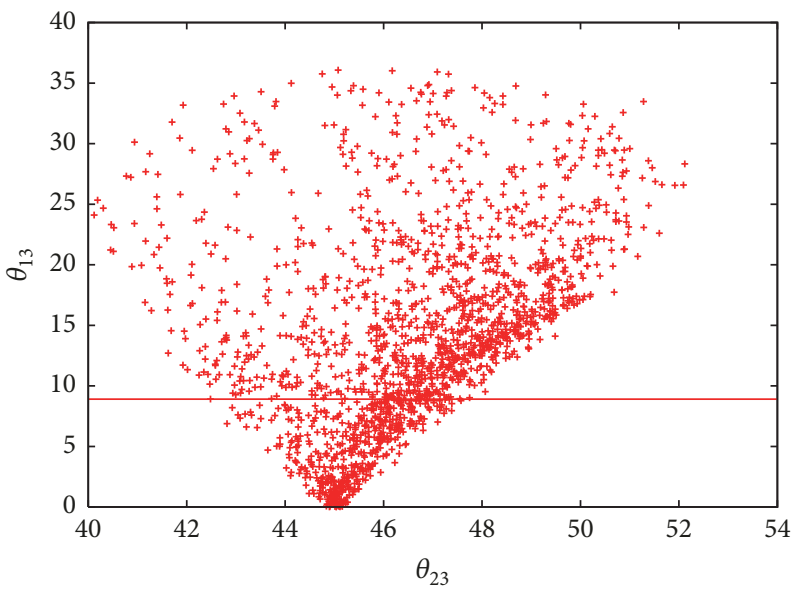

(c)

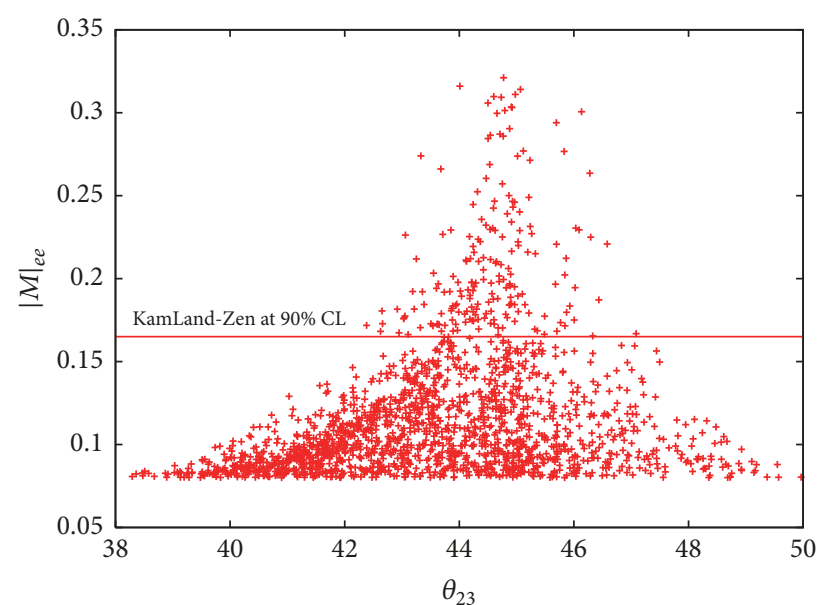

(b)

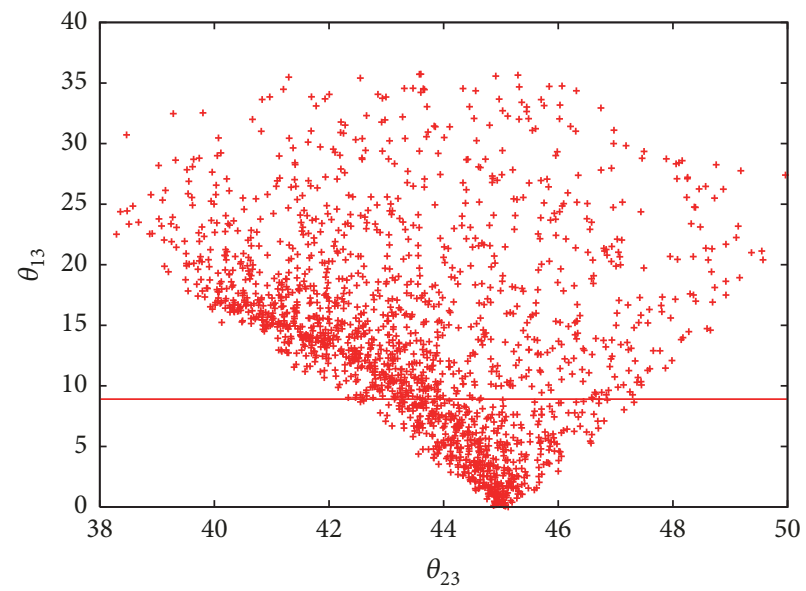

(d)

FIGURE 6: Correlation plots for textures $D_{3}((\mathrm{a}),(\mathrm{c}))$ and $F_{4}((\mathrm{~b}),(\mathrm{d}))$ with NO for 1TEC at $3 \sigma \mathrm{CL}$. The symbols have their usual meaning. In (a) and (b), colored horizontal line indicates the upper limit on effective neutrino mass term $|M|_{e e}$ (i.e., $\left.|M|_{e e}<0.165 \mathrm{eV}\right)$ at $90(<2 \sigma) \% \mathrm{CL}$, given in KamLAND experiment [7]. In (c) and (d), we have shown the upper limit on reactor mixing angle $\theta_{13}$.

for 1TEC with current oscillation data, while normal mass ordering (NO) is ruled out for these cases. For 1TEE, $\rho, \sigma, \delta$ remain unconstrained; however, for 1TEC, only $\delta$ remains unconstrained, while Majorana phases $(\rho, \sigma)$ are restricted near $0^{0}$ pertaining to viable cases. From Table 3 , it is clear that lower bound on lowest neutrino mass $\left(m_{1}(\mathrm{NO})\right.$ or $\left.m_{3}(\mathrm{IO})\right)$ is nearly equal or less than $1 \mathrm{meV}$ for 1TEE and 1TEC.

Category B (C). In Category B, all the ten possible cases are allowed for both 1TEE and 1TEC, respectively, at $3 \sigma$ CL; however, cases $B_{1,6,7}$ allow only NO for 1TEE, while the same allow only IO for 1TEC (Table 4). Cases $B_{2,3,4,5,8,9,10}$ allow both $\mathrm{NO}$ and IO for 1TEE and 1TEC, respectively. As mentioned in $[26,27]$, cases of Category $B$ are related to the cases belonging to Category $\mathrm{C}$ through permutation symmetry; therefore, we can obtain the results for Category $\mathrm{C}$ from $\mathrm{B}$. We find that cases $C_{1,2,3}$ allow only NO for 1TEE, while the same allow IO for 1TEC.

Textures $B_{1}(\mathrm{NO}), B_{3}(\mathrm{NO}, \mathrm{IO}), B_{5}(\mathrm{NO}, \mathrm{IO}), B_{6}(\mathrm{NO}), B_{7}$ $(\mathrm{NO}), B_{8}(\mathrm{NO}), B_{10}(\mathrm{NO}), C_{1}(\mathrm{NO}), C_{2}(\mathrm{NO}), C_{3}(\mathrm{NO}), C_{4}$
(NO, IO), $C_{6}(\mathrm{NO}, \mathrm{IO}), C_{8}(\mathrm{NO})$, and $C_{10}(\mathrm{NO})$ held nearly no constraint on Dirac CP violating phase $(\delta)$ for 1TEE and ITEC, respectively, but with opposite neutrino mass ordering (Table 4 ). Only cases $B_{2}(\mathrm{NO}), B_{4}(\mathrm{IO}), C_{7}(\mathrm{NO})$, and $C_{5}(\mathrm{IO})$ for 1TEE and $B_{2}(\mathrm{IO}), B_{4}(\mathrm{NO}), C_{7}(\mathrm{IO})$, and $C_{5}(\mathrm{NO})$ for ITEC show significant reduction in the parameter space of $\delta$. It is found that $\delta$ is restricted near $90^{\circ}$ and $270^{\circ}$ for 1TEE and 1TEC, respectively (Table 4 ). These predictions are significant considering the latest hint on $\delta$ near $270^{\circ}[5,6]$. Therefore all the above cases discussed are almost indistinguishable for ITEE and 1TEC, if neutrino mass ordering is not considered.

Category $D(F)$. All the ten possible cases belonging to Category D are acceptable with neutrino oscillation data at $3 \sigma$ CL for 1TEE and 1TEC, respectively (Table 5). However cases $D_{1,2,4,5,6,7,9}$ favor both NO and IO for 1TEE and 1TEC, while $D_{3}, D_{8}$, and $D_{10}$ are acceptable only for IO in case of ITEE; however, the same cases allowed NO in case of 1TEC. Similarly, the results for cases belonging to Category F can be derived from Category $\mathrm{D}$. 
TABLE 2: Sixty phenomenologically possible hybrid texture structures of $M_{v}$ at $3 \sigma$ C.L where the triangles " $\triangle$ " denote equal and nonzero elements (or cofactors) and " 0 " denotes the vanishing element (or minor). " $\times$ " denotes the nonzero elements or cofactor.

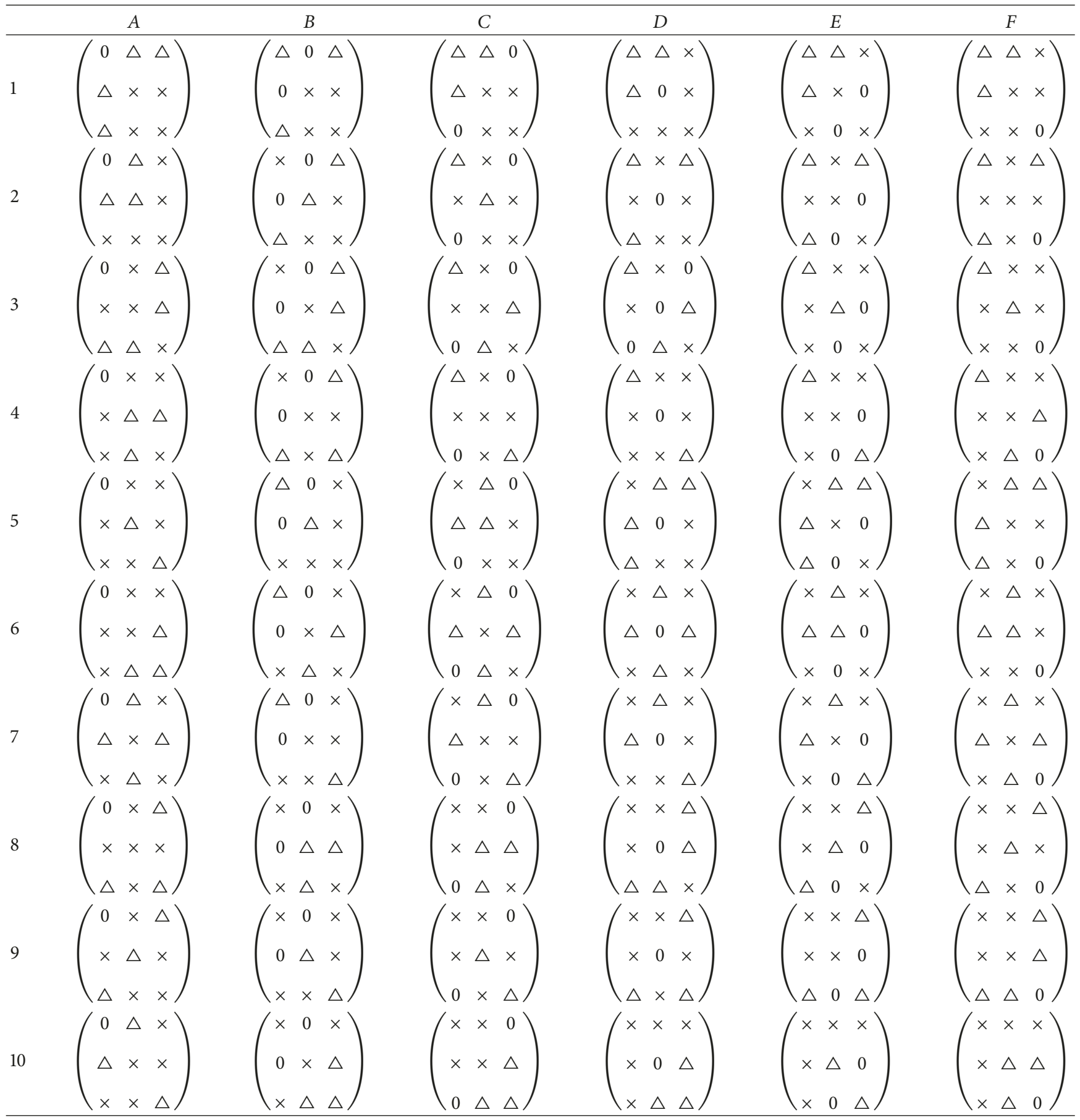

Cases $D_{1}$ (IO), $D_{2}$ (IO), $D_{3}(\mathrm{IO}), D_{4}(\mathrm{IO}), D_{5}(\mathrm{NO}, \mathrm{IO})$, $D_{6}(\mathrm{IO}), D_{7}(\mathrm{NO}, \mathrm{IO}), D_{8}(\mathrm{IO}), D_{9}(\mathrm{NO}, \mathrm{IO}), D_{10}(\mathrm{IO}), F_{1}$ (IO), $F_{2}(\mathrm{IO}), F_{3}(\mathrm{IO}), F_{4}(\mathrm{IO}), F_{5}(\mathrm{NO}, \mathrm{IO}), F_{6}(\mathrm{IO}), F_{7}(\mathrm{NO}$, $\mathrm{IO}), F_{8}(\mathrm{IO}), F_{9}(\mathrm{NO}, \mathrm{IO})$, and $F_{10}(\mathrm{IO})$ predict literally no constraints on $\delta$ for 1TEE. These cases give identical predictions for 1TEC, but for opposite mass ordering. For cases $D_{1}(\mathrm{NO}), D_{2}(\mathrm{NO}), D_{4}(\mathrm{NO}), D_{6}(\mathrm{NO}), F_{1}(\mathrm{NO})$,
$F_{2}(\mathrm{NO}), F_{4}(\mathrm{NO})$, and $F_{6}(\mathrm{NO})$, the parameter space of $\delta$ is found to be well constrained for 1TEE. These cases give similar predictions regarding the parameter space of $\delta$ for ITEC, but for IO.

Category E. In Category E, all the ten possible cases are allowed for 1TEE at $3 \sigma \mathrm{CL}$, while only nine other than $E_{5}$ are 
TABle 3: The allowed ranges of Dirac CP-violating phase $\delta$, the Majorana phases $\rho, \sigma$, and three neutrino masses $m_{1}, m_{2}, m_{3}$ for the experimentally allowed cases of Category A. Masses are in $\mathrm{eV}$. “ $x$ ” denotes the nonviability of case for a particular mass ordering.

\begin{tabular}{|c|c|c|c|c|}
\hline \multirow{2}{*}{ Cases } & \multicolumn{2}{|l|}{ 1TEE } & \multicolumn{2}{|r|}{ 1TEC } \\
\hline & NO & $\mathrm{IO}$ & NO & $\mathrm{IO}$ \\
\hline \multirow{6}{*}{$A_{1}$} & $\rho=-90^{0}-90^{0}$ & $x$ & $x$ & $\rho=-0.0277^{0}--0.0220^{0} \oplus 0.0214^{0}-0.0274^{0}$ \\
\hline & $\sigma=-90^{0}-90^{0}$ & $x$ & $x$ & $\sigma=-0.0273^{0}-0.0271^{0}$ \\
\hline & $\delta=0^{0}-360^{0}$ & $x$ & $x$ & $\delta=0^{0}-360^{0}$ \\
\hline & $m_{1}=0.00147-0.0106$ & $x$ & $\times$ & $m_{1}=0.0430-0.0534$ \\
\hline & $m_{2}=0.00849-0.0139$ & $x$ & $x$ & $m_{2}=0.0439-0.0541$ \\
\hline & $m_{3}=0.0437-0.0551$ & $\times$ & $\times$ & $m_{3}=0.000904-0.00504$ \\
\hline$A_{2}\left(A_{8}\right)$ & $\times$ & $\times$ & $\times$ & $\times$ \\
\hline$A_{3}\left(A_{7}\right)$ & $\times$ & $x$ & $x$ & $\times$ \\
\hline \multirow{6}{*}{$A_{4}\left(A_{6}\right)$} & $\rho=-90^{0}-90^{0}$ & $x$ & $x$ & $\rho=-0.0275^{0}--0.0222^{0} \oplus 0.0215^{0}-0.0269^{0}$ \\
\hline & $\sigma=-90^{0}-90^{0}$ & $x$ & $x$ & $\sigma=-0.0250^{0}-0.0268^{0}$ \\
\hline & $\delta=0^{0}-360^{0}$ & $\times$ & $\times$ & $\delta=0^{0}-360^{0}$ \\
\hline & $m_{1}=0.00148-0.0106$ & $\times$ & $\times$ & $m_{1}=0.0431-0.0534$ \\
\hline & $m_{2}=0.00850-0.0139$ & $\times$ & $x$ & $m_{2}=0.0439-0.0540$ \\
\hline & $m_{3}=0.0437-0.0551$ & $x$ & $\times$ & $m_{3}=0.000950-0.00504$ \\
\hline \multirow{6}{*}{$A_{5}\left(A_{5}\right)$} & $\rho=-90^{0}-90^{0}$ & $x$ & $x$ & $\times$ \\
\hline & $\sigma=-90^{0}-90^{0}$ & $x$ & $x$ & $x$ \\
\hline & $\delta=0^{0}-360^{0}$ & $\times$ & $\times$ & $\times$ \\
\hline & $m_{1}=0.00163-0.0105$ & $\times$ & $\times$ & $\times$ \\
\hline & $m_{2}=0.00854-0.0137$ & $x$ & $x$ & $x$ \\
\hline & $m_{3}=0.0438-0.0545$ & $\times$ & $x$ & $x$ \\
\hline$A_{9}\left(A_{10}\right)$ & $\times$ & $x$ & $x$ & $x$ \\
\hline
\end{tabular}

acceptable in case of 1TEC (Table 6). Cases $E_{1,2,3,4,6,7,8,9}$ allow only inverted mass ordering (IO) for 1TEE, while the same textures allow only normal mass ordering (NO) for 1TEC. Cases $E_{5}$ and $E_{10}$ allow both NO and IO for 1TEE; however $E_{5}$ is ruled out for both NO and IO for 1TEC at $3 \sigma$ CL. Similar to cases belonging to Category $\mathrm{D}, E_{1,2,3,4,5,6,7,8,9}(\mathrm{IO})$ cover full range of $\rho, \sigma, \delta$ for 1TEE, whereas the same cases (except $E_{5}$ ) give identical predictions for 1TEC, but for NO. For $E_{5}$ (NO) and $E_{10}(\mathrm{NO})$, phases $\rho, \sigma, \delta$ are somewhat restricted at $3 \sigma$ CL for 1TEE, while only for $E_{10}$ (IO), the parameter space of $\rho, \sigma, \delta$ seems to be restricted for 1TEC (Table 6).

To summarize our discussion, we have investigated all the viable cases of 1TEE and 1TEC texture structures in the limit of large effective neutrino mass $|M|_{e e}$. It is found that only four cases are able to produce near maximal atmospheric mixing for 1TEE and 1TEC, respectively. However, the predictions remain true irrespective of the experimental data on solar and reactor mixing angle. The observation also hints towards the indistinguishable feature of 1TEE and 1TEC texture structures, but for opposite mass ordering. In order to depict the indistinguishability, we have carried out a comparative study of 1TEE and 1TEC texture structures using the current experimental data at $3 \sigma$ CL. From our discussion we find that most of the cases belonging to 1TEE and 1TEC are almost indistinguishable as far as the neutrino oscillation parameters are concerned, but with opposite neutrino mass ordering. The indistinguishable nature of 1TEE and 1TEC is more prominent for quasi-degenerate mass ordering. For the cases where lower bound on lowest neutrino mass is very small $(<1 \mathrm{meV})$, there is noticeable deviation in the predictions for 1TEE and 1TEC (Tables 3, 4, 5, and 6). This point is also discussed by Liao et. al. in [31]. In addition, the parameter space of $\delta$ for most of the cases belonging to 1TEE and 1TEC remains unrestricted, while only eight cases show maximal restriction for $\delta$. Since no presently feasible experiment has been able to determine the neutrino mass ordering, therefore, we cannot distinguish 1TEE and 1TEC structures using the present oscillation data. However, the currently running and forthcoming neutrino experiments aimed at distinguishing the mass ordering of neutrinos will test our phenomenological results. Also the ongoing and future neutrinoless double beta decay experiments are capable of measuring $|M|_{e e}$ term, which would, in turn, either confirm or rule out our assumption of large $|M|_{e e}$.

\section{Conflicts of Interest}

The author declares that there are no conflicts of interest regarding the publication of this paper. 


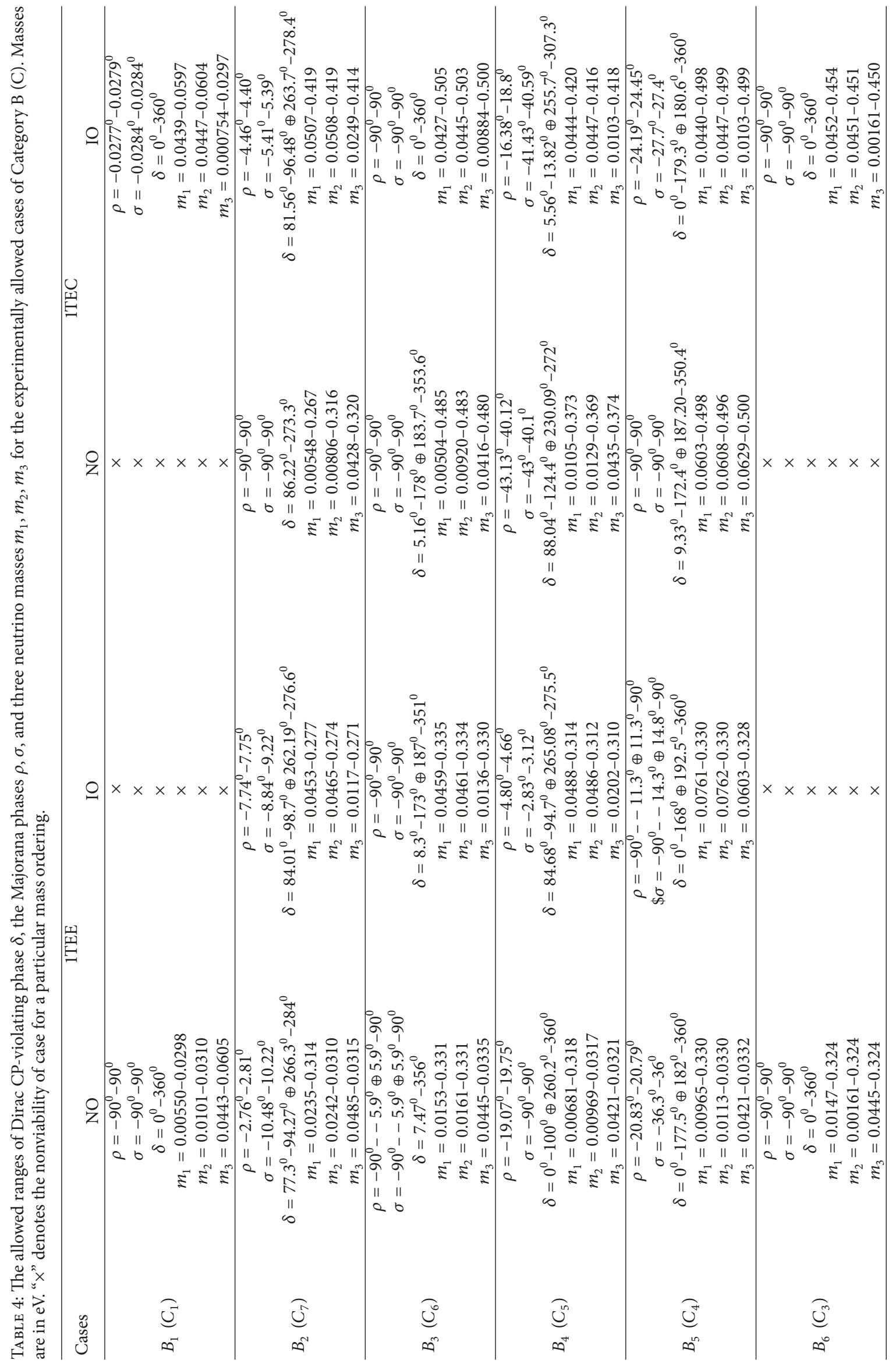




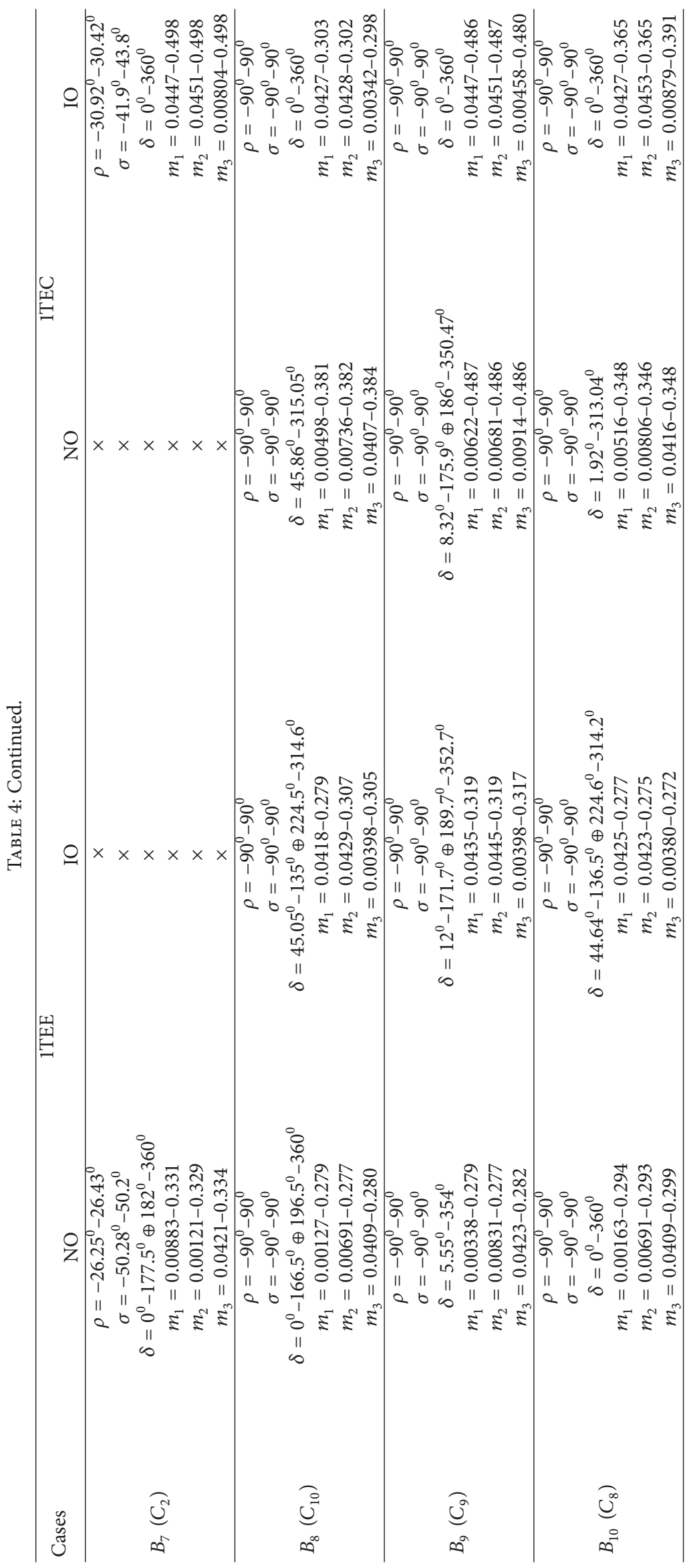




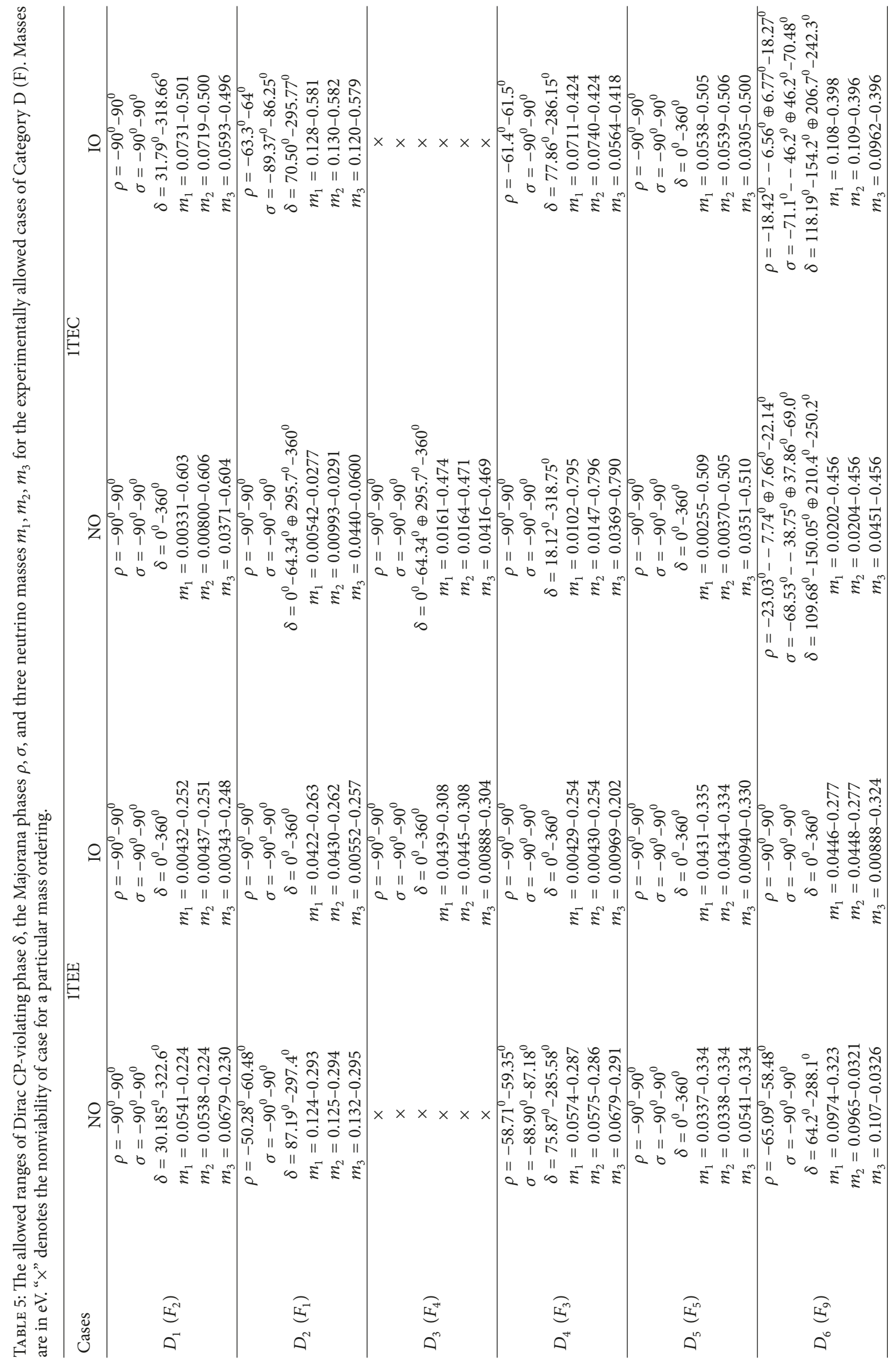




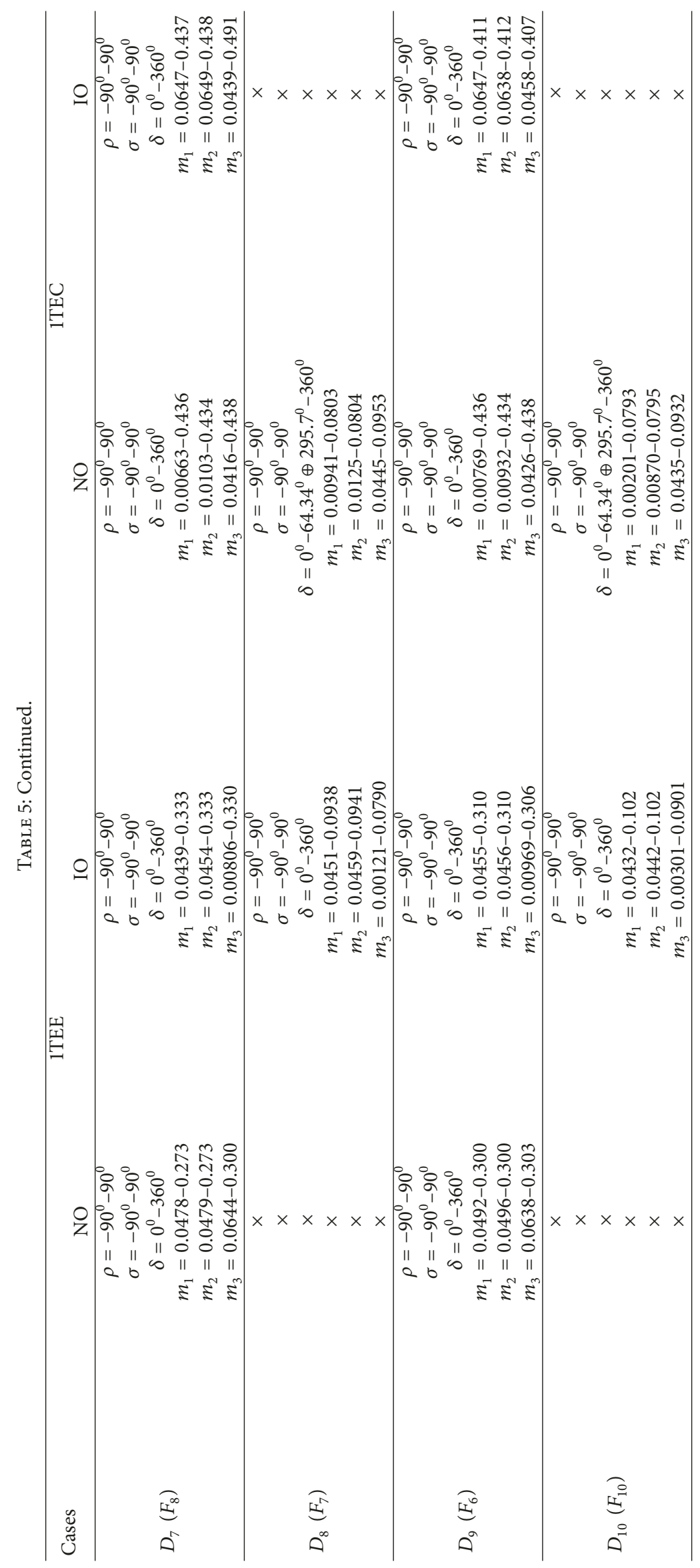




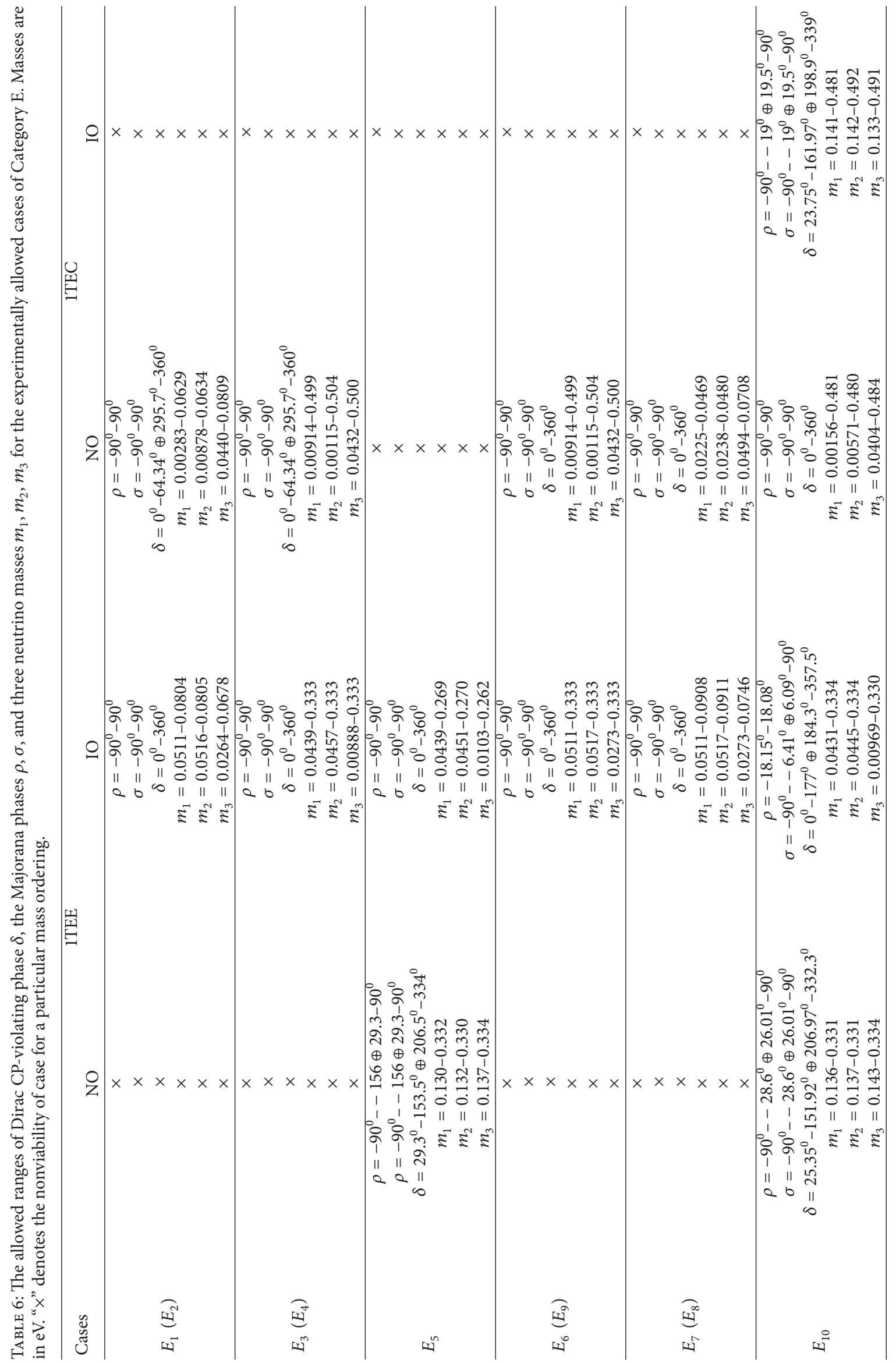




\section{Acknowledgments}

The author would like to thank the Director, National Institute of Technology Kurukshetra, for providing necessary facilities to work.

\section{References}

[1] Y. Abe, C. Aberle, T. Akiri et al., "Indication of Reactor $\bar{v}_{e}$ Disappearance in the Double Chooz Experiment," Physical Review Letters, vol. 108, no. 13, Article ID 131801, 2012.

[2] F. P. An, J. Z. Bai, A. B. Balantekin et al., "Observation of Electron-Antineutrino Disappearance at Daya Bay," Physical Review Letters, vol. 108, no. 17, Article ID 171803, 2012.

[3] J. K. Ahn, S. Chebotaryov, J. H. Choi et al., "Observation of reactor electron antineutrinos disappearance in the RENO experiment," Physical Review Letters, vol. 108, no. 19, Article ID 191802, 2012.

[4] K. Abe, J. Adam, H. Aihara et al., "Observation of Electron Neutrino Appearance in a Muon Neutrino Beam," Physical Review Letters, vol. 112, no. 6, Article ID 061802, 8 pages, 2014.

[5] I. Esteban, M. C. Gonzalez-Garcia, and M. Maltoni, "Updated fit to three neutrino mixing: exploring the accelerator-reactor complementarity," Journal of High Energy Physics, vol. 87, 2017.

[6] P. F. de Salas, D. V. Forero, C. A. Ternes, M. Tortola, and J. W. F. Valle, "Status of neutrino oscillations 2017," https://arxiv.org/ abs/1708.01186.

[7] A. Gando, Y. Gando, T. Hachiya et al., "Search for Majorana Neutrinos Near the Inverted Mass Hierarchy Region with KamLAND-Zen," Physical Review Letters, vol. 117, no. 8, Article ID 082503, 2016.

[8] J. B. Albert, D. J. Auty, and P. S. Barbeau, "Search for Majorana neutrinos with the first two years of EXO-200 data," Nature, vol. 510, pp. 229-234, 2014.

[9] S. Dell'Oro, S. Marcocci, M. Viel, and F. Vissani, "Neutrinoless Double Beta Decay: 2015 Review," Advances in High Energy Physics, vol. 2016, Article ID 2162659, 37 pages, 2016.

[10] W. Rodejohann, "Neutrino-less double beta decay and particlE," International Journal of Modern Physics E, vol. 20, article 1833, 2011.

[11] F. T. Avignone III, S. R. Elliott, and J. Engel, "Double beta decay, Majorana neutrinos, and neutrino mass," Reviews of Modern Physics, vol. 80, article 481, 2008.

[12] J. J. Gómez-Cadenas, J. Martin-Albo, M. Mezzetto, F. Monrabal, and M. Sorel, "The search for neutrinoless double beta decay," La Rivista del Nuovo Cimento, vol. 29, no. 2, 2012.

[13] S. M. Bilenky and C. Giunti, "Neutrinoless double-beta decay: a brief review," Modern Physics Letters A, vol. 27, no. 13, Article ID 1230015, 22 pages, 2012.

[14] P. H. Frampton, S. L. Glashow, and D. Marfatia, "Zeroes of the neutrino mass matrix," Physics Letters B, vol. 536, no. 1-2, pp. 79-82, 2002.

[15] Z.-Z. Xing, "Texture zeros and majorana phases of the neutrino mass matrix," Physics Letters B, vol. 530, no. 1-4, pp. 159-166, 2002.

[16] J. Liao, D. Marfatia, and K. Whisnant, "Texture and cofactor zeros of the neutrino mass matrix," Journal of High Energy Physics, vol. 13, 2014.

[17] D. Meloni, A. Meroni, and E. Peinado, "Two-zero Majorana textures in the light of the Planck results," Physical Review D, vol. 89, no. 5, Article ID 053009, 2014.
[18] P. O. Ludl and W. Grimus, "A complete survey of texture zeros in the lepton mass matrices," Journal of High Energy Physics, vol. 9, 2014.

[19] S. Dev, R. R. Gautam, and L. Singh, "Neutrino mass matrices with two equalities between the elements or cofactors," Physical Review D: Particles, Fields, Gravitation and Cosmology, vol. 87, Article ID 073011, 2013.

[20] J.-Y. Liu and S. Zhou, "Hybrid textures of Majorana neutrino mass matrix and current experimental tests," Physical Review D: Particles, Fields, Gravitation and Cosmology, vol. 87, no. 9-1, Article ID 093010, 2013.

[21] S. Kaneko, H. Sawanaka, and M. Tanimoto, Journal of High Energy Physics, vol. 73, Article ID 0508, 2005.

[22] L. Lavoura, "Zeros of the inverted neutrino mass matrix," Physics Letters B, vol. 609, no. 3-4, pp. 317-322, 2005.

[23] E. I. Lashin and N. Chamoun, "Zero minors of the neutrino mass matrix," Physical Review D: Particles, Fields, Gravitation and Cosmology, vol. 78, no. 7, Article ID 073002, 2008.

[24] E. I. Lashin and N. Chamoun, "One vanishing minor in the neutrino mass matrix," Physical Review D: Particles, Fields, Gravitation and Cosmology, vol. 80, no. 9, Article ID 093004, 2009.

[25] J. Liao, D. Marfatia, and K. Whisnant, "Texture and cofactor zeros of the neutrino mass matrix," Journal of High Energy Physics, vol. 13, Article ID 1409, 2014.

[26] W. Wang, "Neutrino mass textures with one vanishing minor and two equal cofactors," The European Physical Journal C, vol. 73, article 2551, 2013.

[27] S. Dev, R. R. Gautam, and L. Singh, "Hybrid textures of the right-handed Majorana neutrino mass matrix," Physical Review D: Particles, Fields, Gravitation and Cosmology, vol. 88, Article ID 033008, 2013.

[28] S. Dev, R. R. Gautam, L. Singh, and M. Gupta, "Hybrid textures of the right-handed Majorana neutrino mass matrix," Physical Review D: Particles, Fields, Gravitation and Cosmology, vol. 90, Article ID 013021, 2014.

[29] S. Dev, S. Gupta, R. R. Gautam, and L. Singh, "Near maximal atmospheric mixing in neutrino mass matrices with two vanishing minors," Physics Letters B, vol. 706, no. 2-3, pp. 168-176, 2011.

[30] W. Grimus and P. O. Ludl, "Maximal atmospheric neutrino mixing from texture zeros and quasi-degenerate neutrino masses," Physics Letters B, vol. 700, no. 5, pp. 356-361, 2011.

[31] J. Liao, D. Marfatia, and K. Whisnant, "Dual models of the neutrino mass spectrum," Physical Review D: Particles, Fields, Gravitation and Cosmology, vol. 89, no. 1, Article ID 013009, 2014.

[32] J. Liao, D. Marfatia, and K. Whisnant, "Seesaw mechanism with four texture zeros in the neutrino Yukawa matrix," Physical Review D: Particles, Fields, Gravitation and Cosmology, vol. 87, no. 7, Article ID 073013, 2013.

[33] J. Liao, D. Marfatia, and K. Whisnant, "One diagonal texture or cofactor zero of the neutrino mass matrix," Physical Review D: covering particles, fields, gravitation, and cosmology, vol. 88, no. 3, Article ID 033011, 2013. 

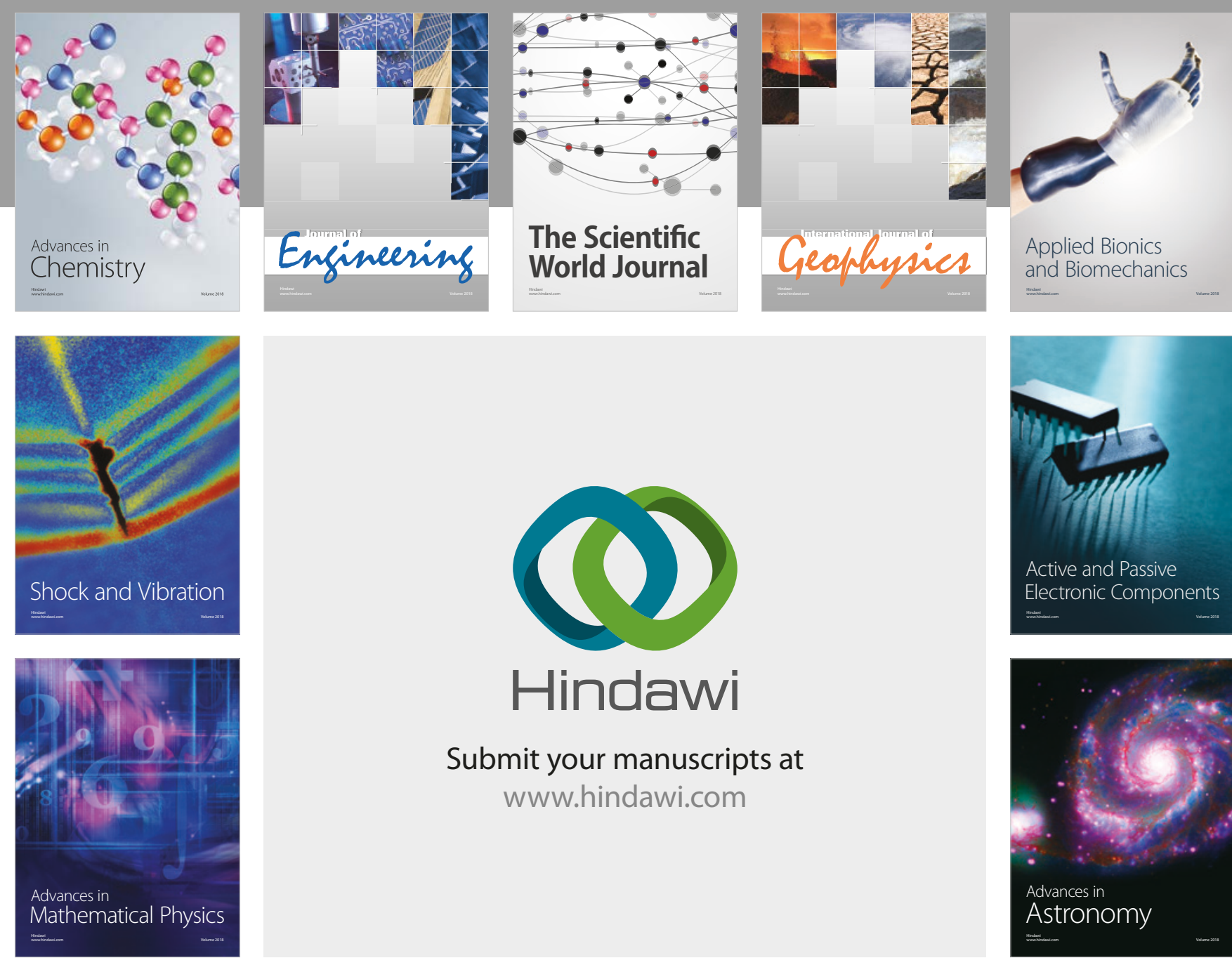

Submit your manuscripts at

www.hindawi.com

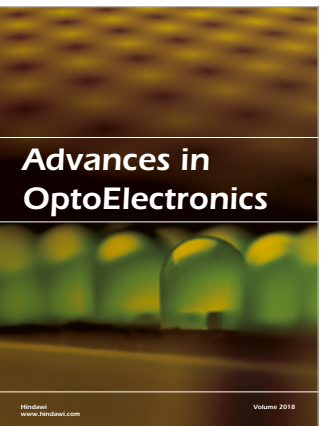

\section{Rotcting Machinery}
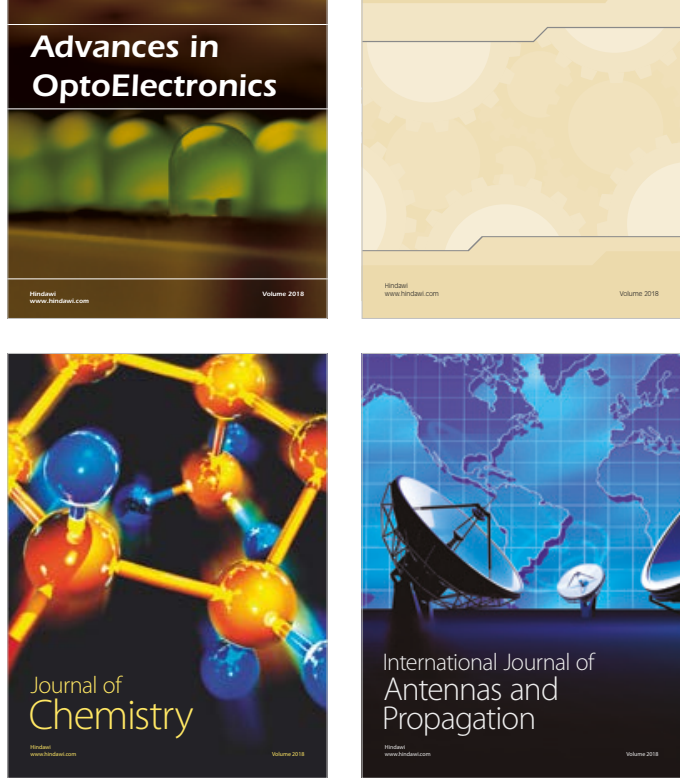

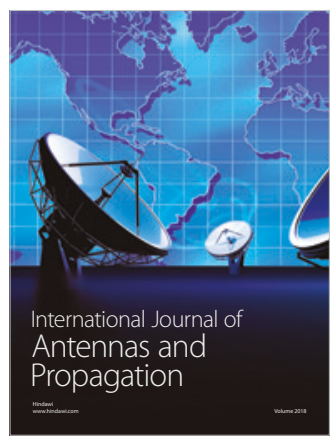

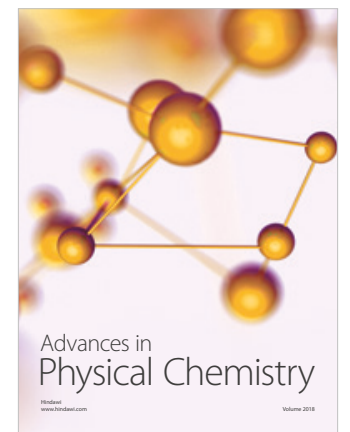

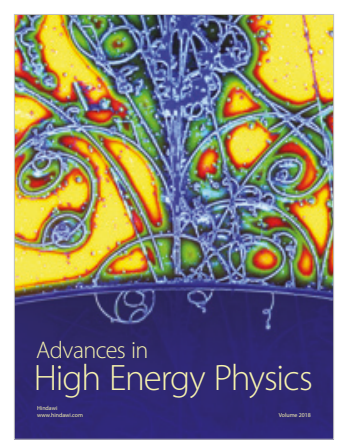

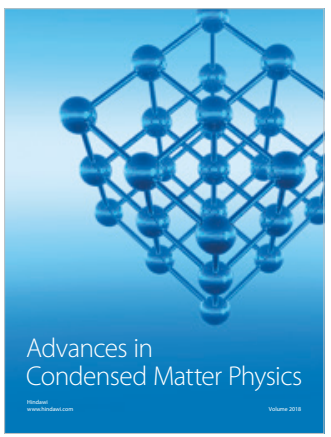

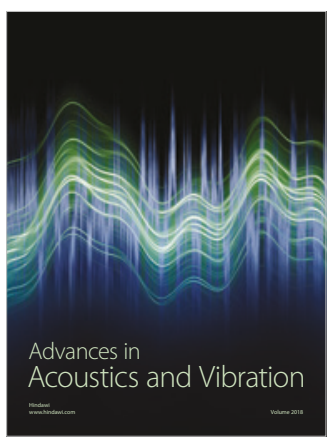

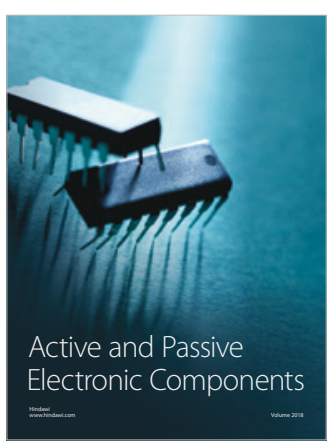
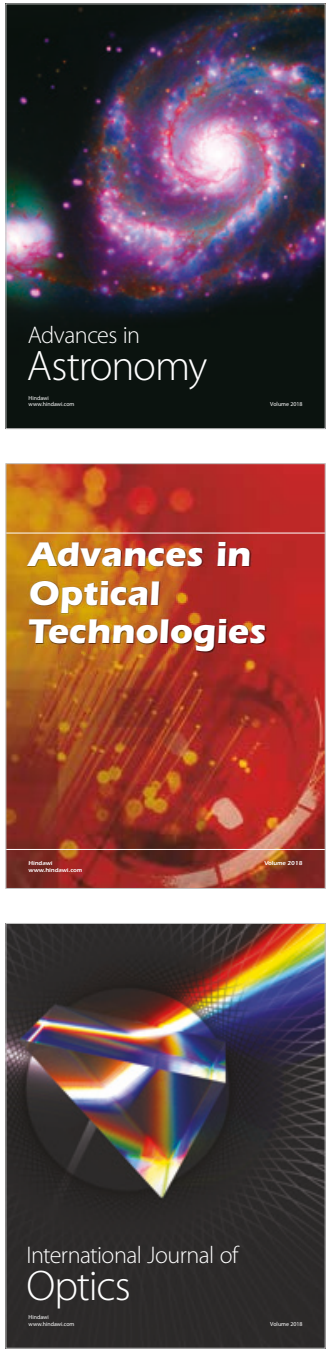